\title{
Emergency Radiology of the Abdomen and Pelvis: Imaging of the Non- traumatic and Traumatic Acute Abdomen
}

\author{
Jay P. Heiken, Douglas S. Katz, and Yves Menu
}

\section{Learning Objectives}

- To distinguish the roles of ultrasound, computed tomography, and magnetic resonance imaging in evaluating traumatic and non-traumatic emergencies of the abdomen and pelvis.

- To discuss the differential diagnosis of acute abdominal and pelvic disorders that present with diffuse abdominal pain and with pain in each abdominal quadrant.

- To evaluate the imaging findings in acute non-traumatic disorders of the abdomen and pelvis.

- To evaluate the imaging findings after blunt or penetrating abdominal trauma.

\subsection{Imaging Techniques}

\subsubsection{General Considerations}

Acute abdominal and pelvic pain is often non-specific, and even under the best of circumstances, physical examination findings and laboratory investigations may not reveal a diagnosis, which is why the radiologist has such a major role in the evaluation of these patients. Because of their substantial

This is the updated version of the chapter by Heiken JP and Katz DS published in: Hodler J, Kubik-Huch RA, von Schulthess GK (Eds), Diseases of the Abdomen and Pelvis 2014-2017, pp. 3-20, Springer 2014.

J. P. Heiken $(\bowtie)$

Mallinckrodt Institute of Radiology, Washington University School of Medicine, St. Louis, MO, USA

e-mail: heikenj@wustl.edu

D. S. Katz · Y. Menu

Hôpital Saint Antoine, Paris, France

e-mail: yves.menu@aphp.fr limitations compared with cross-sectional imaging examinations, abdominal radiographs have a limited role but are still obtained selectively, primarily to evaluate suspected bowel obstruction and/or perforation. Multiple studies have demonstrated the superiority of CT as well as its impact on patient management for imaging the acute abdomen, whether in the trauma or non-trauma setting. Conventional radiographs include supine and upright views, as well as lateral decubitus views. Multi-detector CT, as noted, is the primary imaging examination for evaluation of adult patients with acute abdominal pain, as well as for patients of all ages following acute abdominal and pelvic trauma.

\subsubsection{CT}

The use of CT in patients with an acute abdomen requires careful attention to protocol. In suspected or known urolithiasis, if rupture of an abdominal aortic aneurysm (AAA) is suspected, or if there is a suspected non-traumatic hemorrhage, then generally no intravenous (IV) contrast is administered for initial CT evaluation. In most other cases of non-traumatic acute abdominal and pelvic pain, IV contrast is generally indicated. Typically, $2-3 \mathrm{~mL}$ per second of an appropriate per weight amount of iodinated contrast is administered through a peripheral IV catheter, and portal venous phase images are acquired from the dome of the diaphragm to the inferior aspect of the symphysis pubis, with routine image reconstruction in the transaxial plane using $3 \mathrm{~mm}$ slice thickness and $2 \mathrm{~mm}$ intervals. Thinner sections can be generated in selected cases, but coronal reformations should be created routinely by the CT technologists, sent to PACS, and reviewed by the radiologist. Sagittal (or other) reformations may be helpful in selected cases, especially in patients with bowel obstruction. The data set should be reviewed using routine abdominal windows, bone windows, and lung windows (e.g., to look for extraluminal gas and for pneumothorax at the lung bases in trauma patients). Protocols 
can be modified by the radiologist, in conjunction with the referring physician, for specific situations. For example, a CT angiography protocol can be used for evaluation of suspected bowel ischemia, with thin-section arterial and then routine portal venous phase images acquired.

On post-contrast CT images, hyperattenuating areas can correspond to contrast enhancement or hematoma. Similarly, some calcifications can mimic contrast enhancement. To avoid this ambiguity, some centers recommend acquisition of images using dual energy to allow the reconstruction of virtual unenhanced images whenever necessary. However, the need for dual energy CT acquisition would have to be anticipated, as it is not routinely performed.

In the trauma setting, IV contrast should be given to all patients if possible, but oral contrast is not given (although combined water-soluble oral and rectal contrast may be indicated in selected patients with penetrating trauma, to evaluate for colonic injury). Trauma CT protocols vary depending on the practice and the specific situation and are often combined with CT imaging of other portions of the body. In general a single acquisition is obtained in the late arterial/early portal venous phase, which is then immediately checked by the radiologist. If there are any substantial abnormalities on these images, or if initial plain radiography of the pelvis demonstrates fractures, then a delayed (and ideally relatively low dose) CT acquisition through the area(s) of interest can be performed, to sort out the nature of injuries (i.e., whether a pseudoaneurysm versus an area of active vascular hemorrhage is present; or CT cystography can be performed in suspected bladder injury).

Oral contrast is used less frequently or not at all in an increasing number of practices, for a variety of reasons, in patients undergoing CT for non-traumatic abdominal and pelvic pain. The yield of oral contrast has been questioned, administration of such contrast adds time and minor expense, the contrast does not always reach the distal bowel, and subtle bowel wall pathology may be obscured. However, other practices prefer to give oral contrast for CT of suspected appendicitis and/or when the patient has non-specific abdominal pain. For imaging of suspected solid organ abnormalities, and for suspected urinary tract and gynecologic pathology, oral contrast is not indicated. In suspected highgrade small bowel obstruction or in the setting of mesenteric ischemia or gastrointestinal bleeding, positive oral contrast is contraindicated.

Increased attention has recently been paid to the radiation exposure from diagnostic CT examinations. Although CT appropriately remains the workhorse examination for the acute abdomen, radiation dose reduction strategies should be employed routinely, including the use of mAs reduction and, if available, iterative reconstruction in conjunction with radiation dose reduction (such as lowering the $\mathrm{kVp}$ in thin patients). Multiple CT phases should be acquired only when needed. For patients requiring repeat cross-sectional imaging examinations, alternative strategies, particularly MRI, should be strongly considered whenever feasible.

\subsubsection{Ultrasound}

Ultrasound (US) is the initial imaging examination of choice for patients with suspected acute cholecystitis and acute gynecological abnormalities. It is also the primary method for evaluating pregnant women and pediatric patients with acute abdominal or pelvic pain, in most clinical situations. Although somewhat less sensitive and specific than CT for appendicitis in adult patients, US is a very good first imaging examination when employed by experienced radiologists (and technologists). It also can be used to evaluate the bowel wall (in patients with a body habitus permitting such evaluation) and has a role for rapid triage of trauma patients. A variety of probes and techniques are routinely employed, including Doppler imaging and graded compression for right lower quadrant evaluation.

\subsubsection{Magnetic Resonance Imaging}

Magnetic resonance imaging (MRI) has a growing role in the imaging of pregnant women with abdominal pain who have undergone a nondiagnostic US examination. In a variety of mostly retrospective, small- to medium-sized studies, MRI has been shown to have high accuracy in the evaluation of appendicitis (Fig. 13.1) and has utility for identification and evaluation of alternative conditions, including other bowel

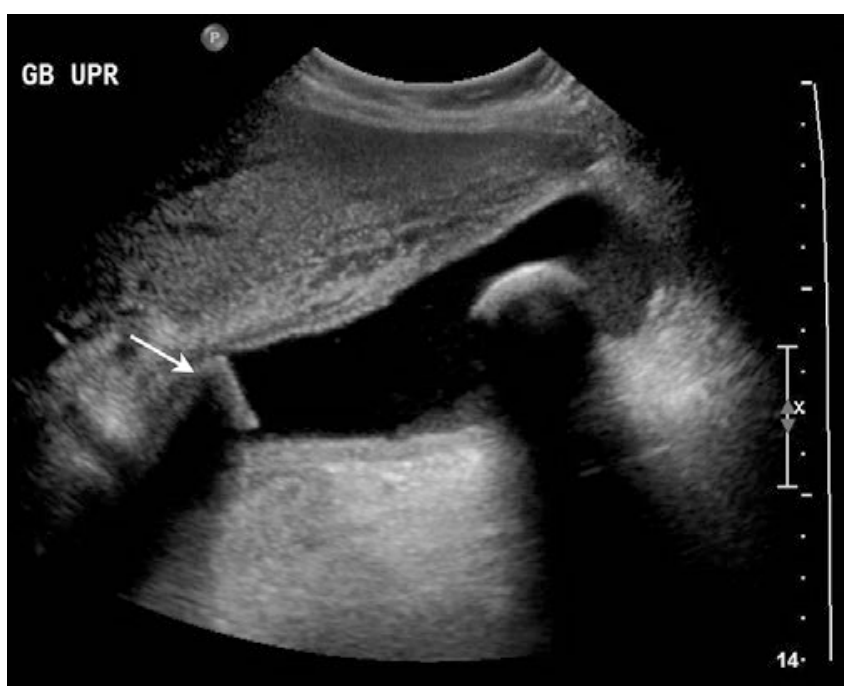

Fig. 13.1 Acute cholecystitis. Ultrasound image demonstrates moderate distention of the gallbladder with wall thickening, sludge, and stones, including one impacted in the gallbladder neck (arrow) 
pathology (such as Crohn disease), and for urinary and biliary tract pathology. Non-contrast sequences are obtained at 1.5 Tesla (or lower), with an emphasis on fast, multi-planar gradient echo/T2-weighted sequences.

\subsection{Acute Pain in an Abdominal Quadrant}

The differential diagnosis in a patient with an acute abdomen is greatly influenced by the nature and location of the pain. Therefore, the imaging strategies localized to each of the four abdominal quadrants will be discussed first, followed by pain which is diffuse or localized to the flank or the epigastric region.

\subsubsection{Right Upper Quadrant}

Pain from gallstones, particularly acute cholecystitis, is by far the most common group of disorders to present with acute right upper quadrant pain. Other differential diagnostic considerations include hepatitis from a variety of etiologies, liver abscess, and rarely a ruptured liver mass (usually hepatocellular adenoma or carcinoma).

US is the imaging examination of choice for evaluation of acute right upper quadrant pain. It is an accurate examination for diagnosing or excluding acute cholecystitis. Sonographic findings include gallstones, a sonographic Murphy's sign, wall thickening of $3 \mathrm{~mm}$ or greater, and pericholecystic fluid/ inflammatory changes (Fig. 13.1). The more these findings are present, particularly gallstones combined with a sonographic Murphy's sign, the more likely acute cholecystitis is present. Isolated gallbladder wall thickening may be due to a variety of etiologies in addition to acute cholecystitis.

Recent publications have shown that CT and MRI also are relatively accurate imaging examinations for cholecystitis, although the findings may be more subtle compared with sonography, especially early on. In our experience, patients with non-specific abdominal pain and the eventual diagnosis of cholecystitis may be sent for CT on a relatively frequent basis rather than for sonography, and radiologists need to be aware of the potential for establishing or suggesting this diagnosis based on the initial CT findings, which are very similar to those on sonography, although pericholecystic inflammatory changes may be more obvious on CT and MRI. If the CT findings suggest cholecystitis but are not definitive, US can then be performed. CT also is an excellent examination for demonstrating complications and severe forms of cholecystitis, including perforation/abscess formation, hemorrhage, gas (in diabetic patients with emphysematous cholecystitis), and wall gangrene. The CT findings of a stone lodged in the gallbladder neck or cystic duct and areas of absent wall enhancement have been shown to correlate with the need for an open rather than a laparoscopic cholecystectomy. CT is also accurate in differentiating cholecystitis from gallbladder cancer, which sometimes is challenging.

US, CT, and MRI are all accurate examinations for the diagnosis (and follow-up) of liver abscesses (Fig. 13.2), which usually are pyogenic but which also may be amebic. US usually demonstrates a round or oval hypoechoic mass or masses, which may contain low-level echoes. Occasionally a hepatic abscess may simulate a solid (or partially solid)
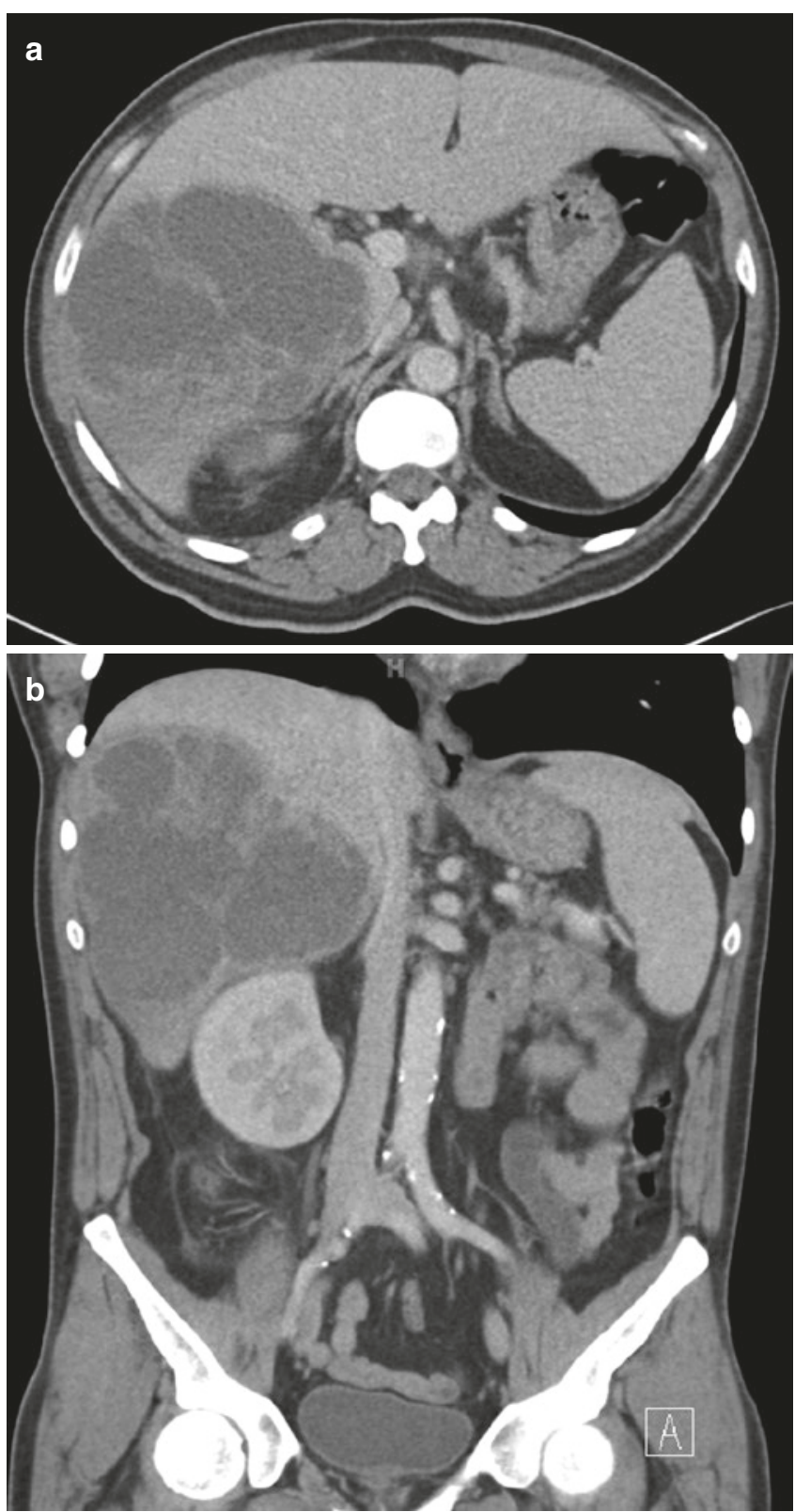

Fig. 13.2 Hepatic abscess. 48-year-old man with right upper quadrant abdominal pain, fever, and leukocytosis. Transaxial (a) and coronal (b) contrast-enhanced CT images demonstrate a large fluid attenuation mass in the right hemiliver with a thick enhancing wall and septations. A drainage catheter was placed, and cultures grew Streptococcus anginosus-constellatus-intermedius group 
hepatic mass on cross-sectional imaging examinations (especially with certain Klebsiella species). Pyogenic liver abscesses may be idiopathic or may result from seeding from infection in the biliary tract, from the luminal gastrointestinal tract or from the portal/mesenteric venous system. An enhancing wall and a peripheral zone of edema are common on CT and MRI, but are not universally present.

Spontaneous rupture of a hepatocellular carcinoma with associated hemoperitoneum is a frequent complication in countries with a high incidence of this tumor, but is very uncommon in Western countries (less than $2 \%$ of cases). Hepatocellular carcinoma usually is highly vascular, and tumor necrosis with associated hepatic capsular rupture and rupture of vessels within the tumor is the presumed etiology. The differential diagnosis includes spontaneous hemorrhage within a hepatic adenoma or a hepatic metastasis. Patients present with acute pain and blood loss. Rapid diagnosis and therapy are essential. Transcatheter embolization is the treatment of choice.

\subsubsection{Left Upper Quadrant}

Although infrequent, acute left upper quadrant pain is most often caused by splenic infarction or splenic abscess (e.g., secondary to bland or septic emboli to the spleen) (Fig. 13.3), but may also occur in the setting of acute gastric disorders including gastritis and ulcer. Generally CT is performed first, although US may occasionally be the first imaging examination in such patients. The diagnosis of gastric pathology is best established by endoscopy, although patients may present with left or right upper abdominal pain, and with other non-specific signs and

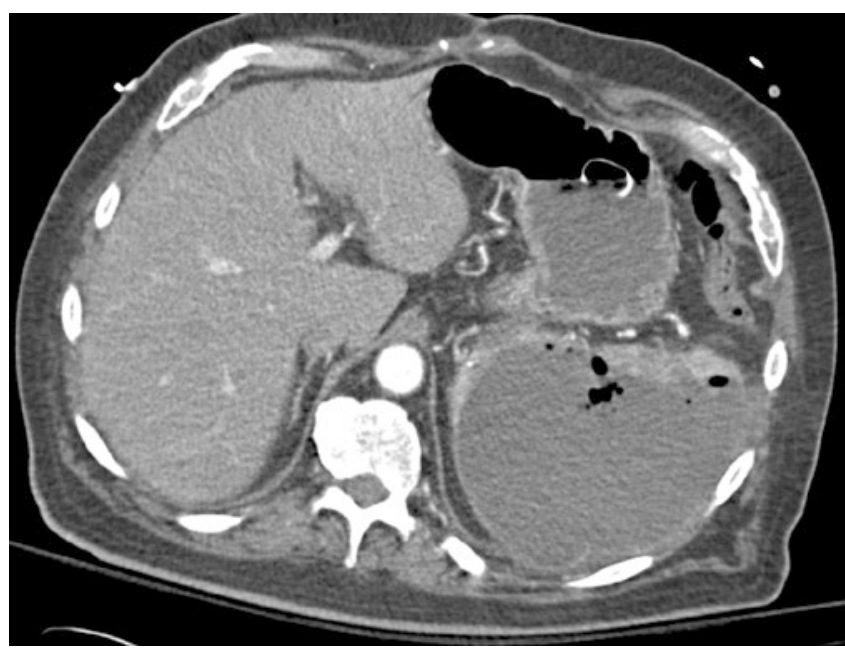

Fig. 13.3 Splenic abscess. 73-year-old man with left upper quadrant pain, fever, and leukocytosis. Transaxial contrast-enhanced CT image shows a gas-containing fluid collection in the spleen. A drainage catheter was placed percutaneously, and cultures grew Escherichia coli symptoms, and CT may reveal the diagnosis when it is not clinically suspected. CT findings include focal or diffuse gastric wall thickening, increased mucosal enhancement, submucosal edema, and perigastric inflammatory changes. Focal ulceration with or without perforation also may be identified, which is generally of benign but occasionally of malignant etiology.

Splenic infarction may be focal and less commonly global. Other etiologies of splenic infarction include marked splenomegaly with outgrowth of the splenic blood supply and pancreatitis. Typical infarcts are wedge-shaped and hypoattenuating on CT. Most splenic abscesses are secondary to hematogenous dissemination of infection and are seen primarily in immunocompromised individuals and IV drug abusers. On CT, splenic abscesses demonstrate low attenuation centrally with an enhancing rim and occasionally have central gas. Spontaneous splenic rupture can occur in patients with splenomegaly caused by hematologic malignancy or viral infection (e.g., mononucleosis).

\subsubsection{Right Lower Quadrant}

Appendicitis is the most frequent cause of acute right lower quadrant pain and the most commonly encountered diagnosis leading to surgery. Other disorders that can present with acute right lower quadrant pain include Crohn disease, rightsided colitis or diverticulitis, and, in women, pelvic inflammatory disease, ovarian cysts (and their complications, including rupture, hemorrhage, and torsion), and other obstetric and gynecologic conditions. Less common causes include omental infarction (which also can present with upper or mid right abdominal pain), epiploic appendagitis, and small bowel diverticulitis (ileal or Meckel's).

Preoperative cross-sectional imaging continues to play an important role in the diagnosis or exclusion of appendicitis, as well as in the identification of complications or alternative diagnoses. At most centers, even when the clinical presentation is typical (in up to one-third of patients, it is not), CT usually is obtained to confirm the diagnosis and to serve as a surgical road map (Fig. 13.4). The most specific CT finding is a thick-walled, dilated, fluid-filled appendix (occasionally containing gas), which may have one or more liths. Contrastenhanced CT often demonstrates "stratification," in which the individual layers of the appendiceal wall can be identified, with mucosal hyperenhancement and submucosal edema. As the disorder progresses, there is increasing inflammation of the adjacent fat, especially in the retroperitoneal planes. Findings indicative of perforation include periappendiceal abscess, extraluminal gas or lith(s), a defect in the appendiceal wall, and associated small bowel obstruction. Determination of the severity of appendicitis is increasingly important as treatment strategies continually evolve. Surgery 

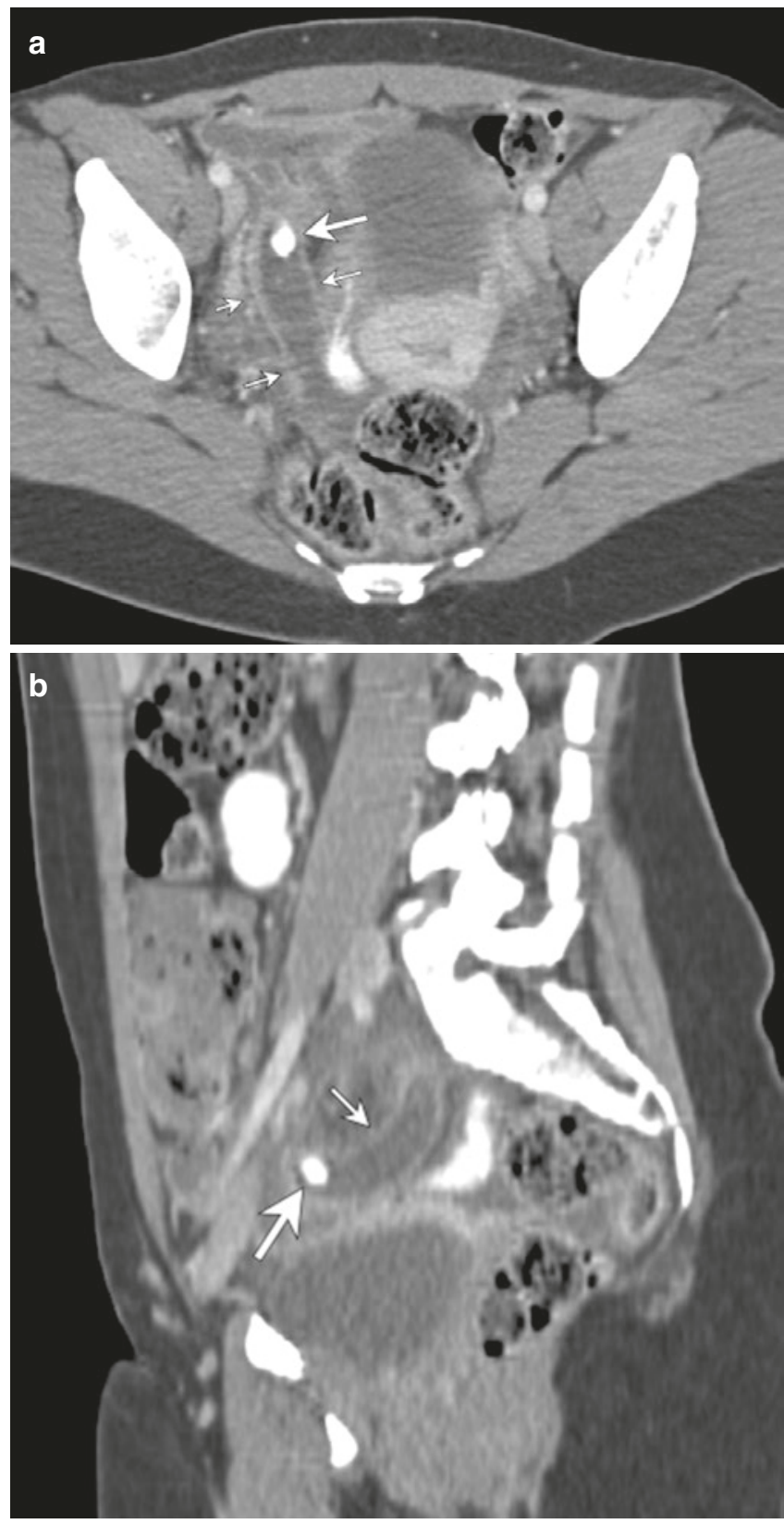

Fig. 13.4 Acute appendicitis. Transaxial (a) and sagittal (b) contrastenhanced CT images demonstrate a dilated, thick-walled, fluid-filled appendix (small arrows) with an appendicolith (large arrow) at the appendiceal orifice

should be performed emergently for severe forms of appendicitis, but alternative options that are increasingly used at some centers include delayed surgery, either the next day or on an outpatient basis, and medical treatment with imaging follow-up.

Opacification of the entire appendiceal lumen is a helpful finding to exclude appendicitis, if oral contrast is administered; however, as noted, some institutions/practices have eliminated the routine use oral contrast for CT of the acute abdomen. Regardless of the specific CT protocol used, interpretation depends in part on the experience of the interpreting radiologist. In a minority of cases, particularly in early and subacute/chronic appendicitis, the imaging findings can be subtle. It should be noted that the appendix can be larger than $6 \mathrm{~mm}$ in transverse diameter and can still be normal on CT, if other findings of appendicitis are absent. In the evaluation of suspected appendicitis in children (Fig. 13.5) (and in selected nonpregnant women of childbearing age), US is considered the initial examination of choice, if available, although CT has a higher accuracy. Demonstration of a swollen, non-compressible appendix $>7 \mathrm{~mm}$ in diameter, with a target configuration (and with pain corresponding to the site of the appendix), is the primary US criteria. An appendicolith or multiple appendicoliths may be identified.

In pregnant women, appendicitis has a more variable presentation, and diagnosis is more challenging with all crosssectional imaging modalities. US remains the initial imaging examination, despite its poor sensitivity and specificity in this clinical setting. Non-contrast MRI (Fig. 13.6) is being used increasingly in such patients after nondiagnostic US and has demonstrated high accuracy for diagnosis and exclusion of appendicitis and for identification of alternative diagnoses. CT should be reserved as a third-line examination, used only if needed.

A potential pitfall of image interpretation in patients with suspected acute appendicitis is the occurrence of "secondary appendicitis." In patients with adjacent inflammatory processes such as enteritis or pelvic inflammatory disease, the appendix may be inflamed secondarily as an extension of the regional process. Differentiation between secondary and primary appendicitis may be difficult as some thickening of adjacent bowel loops, including the terminal ileum, may occur in cases of "primary appendicitis."

The CT findings of right-sided diverticulitis are similar to those of the more common left-sided diverticulitis, including an inflamed diverticulum, adjacent diverticula, colonic wall thickening, and pericolonic inflammatory changes. Localized inflammation may be substantial. When considering a diagnosis of cecal diverticulitis, it is important to identify a normal appendix. Microperforation may occur, but more substantial complications, which are common in left-sided diverticulitis, including abscess formation, fistula, and obstruction, are highly unusual in right-sided diverticulitis.

Crohn disease often is first evaluated with CT; however, because patients with known Crohn disease usually are young and require serial imaging, follow-up imaging with MR enterography has become increasingly important to reduce radiation exposure (Fig. 13.7). Cross-sectional imaging permits evaluation of wall thickening and enhancement; changes of the adjacent fat, vessels, and lymph nodes; and assessment of complications, including formation of abscesses, strictures, and fistulas. 

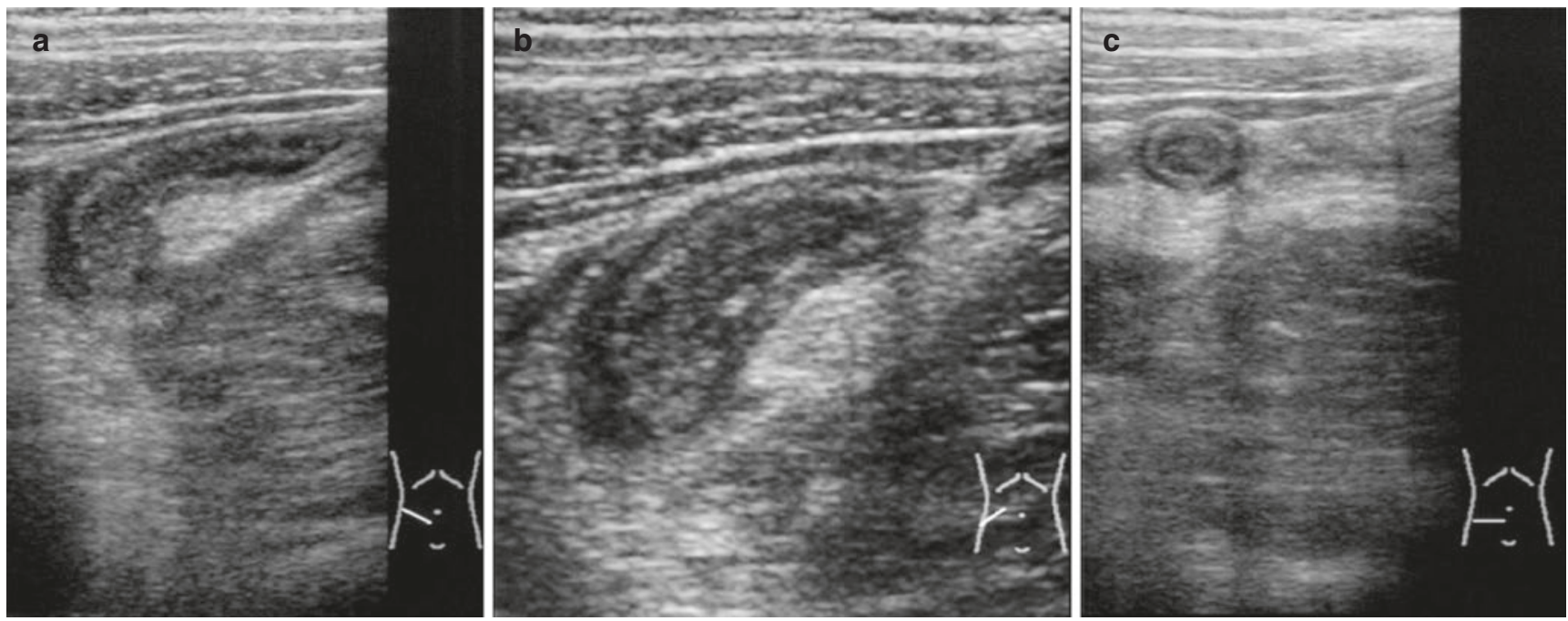

Fig. 13.5 (a-c) Ultrasonography of acute appendicitis in a 12-year-old girl. Oblique and transverse views show a swollen appendix (diameter $10 \mathrm{~mm}$ ), with a target configuration

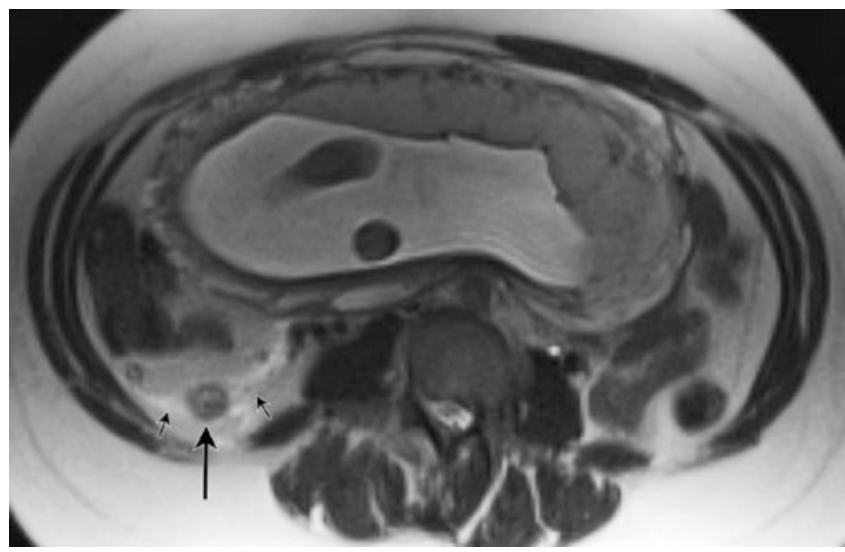

Fig. 13.6 Acute appendicitis. 30-year-old woman who is 21 weeks pregnant with right lower quadrant pain. Transaxial T2-weighted unenhanced MR image shows a thick-walled retrocecal appendix (large arrow) with surrounding inflammation (small arrows)

\subsubsection{Left Lower Quadrant}

Diverticulitis of the sigmoid or descending colon is a very common disorder in middle-aged and older patients, but also can affect young individuals. CT is the examination of choice, which is typically performed with IV contrast, with or without oral or rectal contrast. Other protocols include non-contrast CT. In uncomplicated disease, the CT (or MR) findings are the same as with right colonic diverticulitis. Patients with redundancy of the sigmoid colon may present with midline or right lower quadrant pain. CT also demonstrates complications, including microperforation/extraluminal gas (Fig. 13.8), fistula/abscess formation, and unusual complications such as venous thrombosis, liver abscess, urinary tract obstruction, and adnexal involvement. Recent data suggest that only selected patients identified with what is believed to be left colonic diverticulitis on CT require follow-up optical colonoscopy to exclude underlying carcinoma (i.e., those with $\mathrm{CT}$ findings that are atypical and/or complicated).

Other diagnostic considerations in patients presenting with left lower quadrant pain include colitis of a variety of etiologies, including infection (especially from $C$. difficile, in the correct clinical setting) and ischemia; functional colonic disorders (especially constipation and also obstruction from a variety of other etiologies); urinary tract infection; and gynecologic disease.

Epiploic appendagitis is a relatively common, self-limiting condition, in which an appendage of fat along the external aspect of the colon, left side much more common than right, undergoes torsion, with subsequent venous thrombosis. CT findings include a swollen, ovoid, $1.5-3.5 \mathrm{~cm}$ fat-containing focus with peripheral thickening and associated inflammation. The adjacent colon is usually normal or nearly normal.

\subsection{Gynecologic Disorders}

US is the primary imaging examination in females with suspected gynecologic pathology. A human chorionic gonadotropin (HCG) level should be checked emergently in all women of childbearing age with acute non-traumatic abdominal and pelvic pain to exclude ectopic or incidental pregnancy. MRI is being used increasingly as a problem-solving examination after pelvic US and 

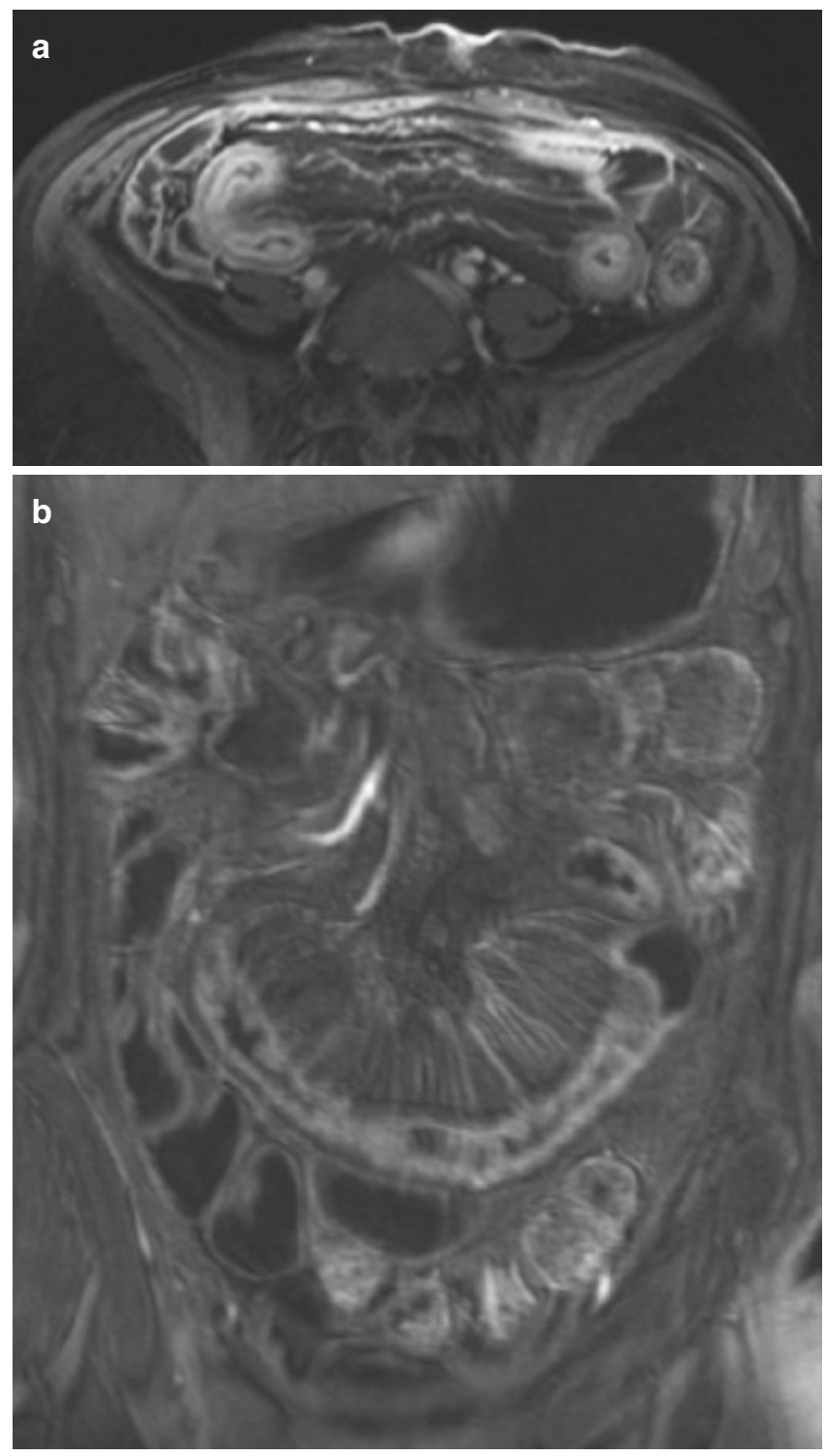

Fig. 13.7 Crohn disease. 55-year-old woman with abdominal pain, diarrhea, and gastrointestinal bleeding. Transaxial (a) and coronal (b) contrast-enhanced T1-weighted images with fat suppression demonstrate ileal wall thickening, mucosal hyperenhancement, submucosal edema, and mesenteric vascular engorgement indicative of disease flair

for follow-up. However, because women with acute gynecologic disorders often present with non-specific signs and symptoms, $\mathrm{CT}$ is frequently ordered to evaluate their abdominal pain. Therefore radiologists interpreting CT of the acute abdomen and pelvis must be familiar with the findings of a wide variety of gynecologic disorders that may present emergently. CT also can be used selectively as a problem-solving tool in patients with known or suspected gynecologic disorders.

If ovarian torsion is suspected prospectively, pelvic US is performed emergently. However, CT and MR findings also are characteristic, are similar to US findings, and may complement
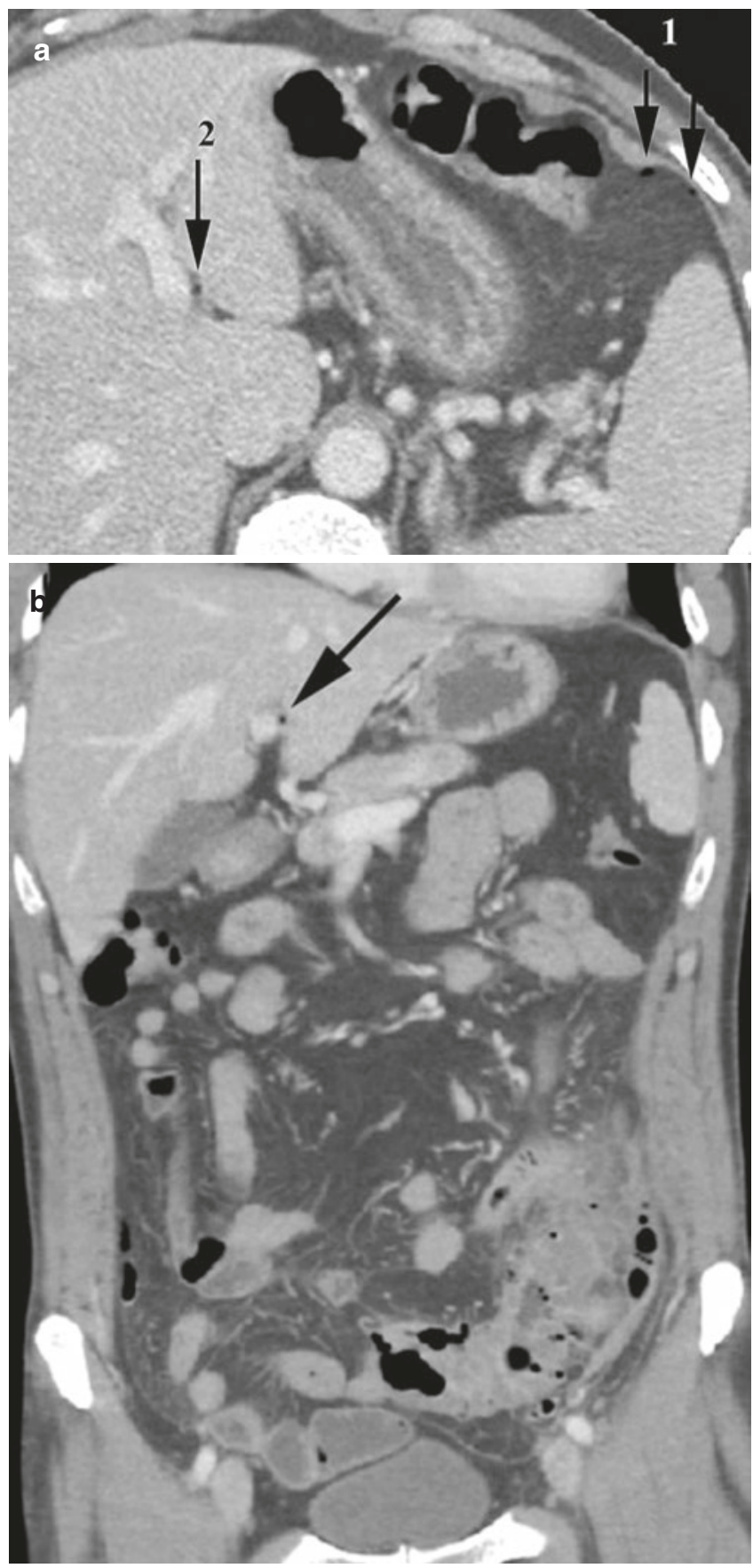

Fig.13.8 (a, b) Perforated sigmoid diverticulitis. (a) Axial CT image demonstrates tiny bubbles of extraluminal gas within the left subphrenic space (arrows, 1) and hepatic pedicle (arrow, 2). (b) Coronal CT reformation shows thickened sigmoid colon, with associated diverticular disease, inflammation of the adjacent fat, and thickening of the root of the sigmoid mesocolon. Note the tiny bubble of extraluminal gas (arrow) within the hepatic pedicle

US if the sonographic examination is initially equivocal. Radiologists must maintain a high index of suspicion for ovarian torsion as the clinical presentation is variable; the possibility of torsion should be raised if the imaging findings suggest 
or strongly point to this diagnosis. Cross-sectional imaging findings include an enlarged, edematous ovary, with or without an associated cyst or mass (most commonly a teratoma), deviation of the adnexal structures to the contralateral side, deviation of the uterus to the side of torsion, twisting of the adnexal/vascular structures, decreased ovarian vascularity, multiple peripheral ovarian follicles, inflammation of the adjacent fat, and ascites which may be high attenuation due to blood.

Ovarian cysts are very common and may be complicated by hemorrhage. Associated hemoperitoneum, if present, usually is self-limited. The differential diagnosis of a hemorrhagic ovarian cyst is endometrioma. It is particularly critical to check the HCG level in a woman with otherwise unexplained hemoperitoneum, to ensure that a ruptured ectopic pregnancy is not present.

Pelvic inflammatory disease (PID) is common and has a broad spectrum of findings and presentations. If PID is known or suspected, US is done first. However, CT may be initially performed if the diagnosis is not known and the clinical presentation is non-specific (Fig. 13.9). The most common causative organisms are gonococcus and chlamydia. Because PID most commonly results from ascending infection, it usually involves both adnexa. In the earlier stages of PID, there may be no findings or subtle findings on cross-sectional imaging. There may be swelling of the ovaries and/or fallopian tubes, abnormal enhancement of the adnexal structures, and mild adjacent edema. In later stages, tubo-ovarian abscesses and/or hydrosalpinx/pyosalpinx may be identified, and adjacent organs also may be involved in complicated cases. Imaging findings include thickening and increased enhancement of the dilated, tortuous fallopian tube(s), a septated mass in the ovary or ovaries, and adjacent edema. Other presentations of PID include endometritis, cervicitis, and Fitz-Hugh-Curtis syndrome in which perihepatitis leads to increased enhancement of the liver edge.

Other acute gynecologic disorders that can be identified on cross-sectional imaging examinations include less common forms of PID (e.g., actinomycosis), endometritis, ovarian vein thrombosis (typically following cesarean section), uterine rupture following delivery, gynecologic tumors, and complicated uterine leiomyomas (e.g., hemorrhage/degeneration, torsion, and acute prolapse).

\subsection{Acute Abdomen with Diffuse Pain}

Any disorder that involves a large portion of the gastrointestinal tract or irritates the peritoneum can cause diffuse abdominal pain. The most common disorder is gastroenteritis, in which the CT findings often are normal or may show mild bowel wall thickening and increased intraluminal fluid. Patients with colitis have varying degrees of colonic wall thickening/edema, with inflammation of the adjacent fat. Other important disorders that present with diffuse abdominal pain include bowel obstruction and ischemia.
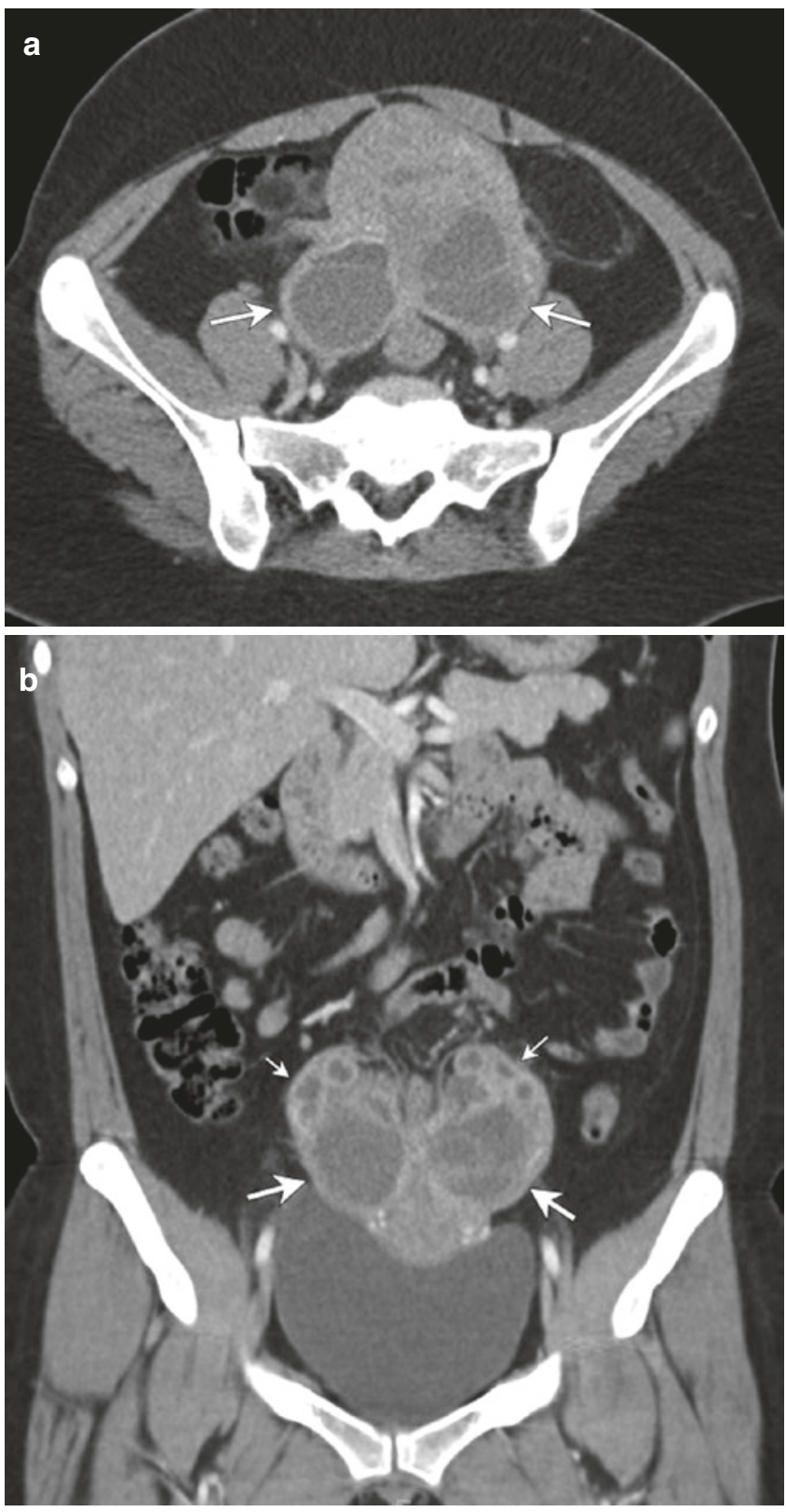

Fig.13.9 Pelvic inflammatory disease. 27-year-old woman with lower abdominal pain, fever, and leukocytosis. Transaxial (a) and coronal (b) contrast-enhanced CT images show hydrosalpinx (small arrows) and a thick-walled fluid collection (large arrows) in both adnexa

\subsubsection{Bowel Obstruction}

Bowel obstruction is a very frequent cause of abdominal pain in the emergency setting. Small bowel obstruction (SBO) usually is due to adhesions (more commonly in patients with previous surgery, but occasionally in patients without prior surgery) and less commonly due to a hernia (external or internal), obstructing tumor, perforated appendicitis, inflammatory bowel disease, or complicated diverticulitis. Less common etiologies include gallstone ileus, intussusception, intramural small bowel hematoma, and radiation enteritis. 
Large bowel obstruction (LBO) is most commonly due to colorectal carcinoma, but diverticulitis and sigmoid or cecal volvulus also are important etiologies.

Bowel obstruction usually is diagnosed on clinical grounds and then confirmed and further evaluated as needed by imaging, typically with $\mathrm{CT}$, with or without initial plain radiographs. However, not infrequently the diagnosis of bowel obstruction is not clear or known clinically, and obstruction is established on the basis of imaging. CT reveals the presence or absence of obstruction, as well as the site, level, and cause of obstruction, and permits identification of associated ischemia (Fig. 13.10). In SBO, the radiologist should search for the transition zone between the dilated proximal small bowel and the collapsed distal small bowel. There often is a segment of small bowel containing feceslike material, just proximal to the transition zone. Review of multi-planar CT reformations is important to help identify the site of transition, which may be difficult to find conclusively on review of transaxial images alone. In a minority of cases, CT reveals a closed-loop SBO (Fig. 13.10), which usually requires emergency surgery. In this situation, two points along the course of the small bowel are obstructed at a single site, usually associated with an adhesive band or hernia. Less commonly, closed-loop SBO may be due to volvulus of the small bowel.

SBO needs to be differentiated from LBO and from ileus and motility disorders involving the small and/or large bowel. Ileus of the small and/or large bowel is common after abdominal surgery and has multiple etiologies. In LBO, CT demonstrates distension of the colon to the point of obstruc-

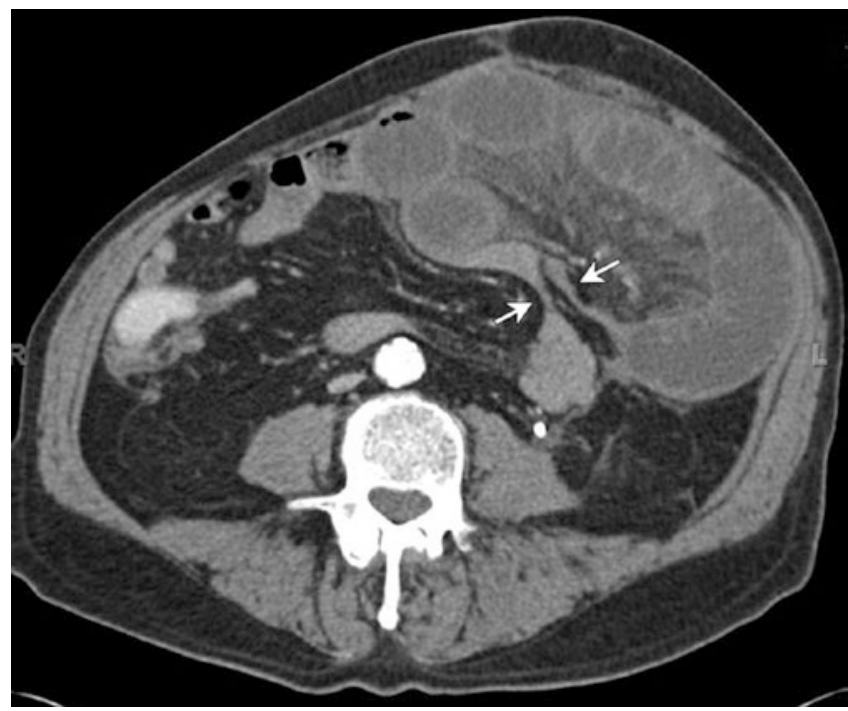

Fig. 13.10 Closed-loop small bowel obstruction. 62-year-old man with metastatic colon cancer who presented with severe abdominal pain, nausea, and vomiting. Transaxial contrast-enhanced CT image shows a fluid-filled, distended loop of small bowel in the left midabdomen. Mesenteric edema and mild wall thickening indicate bowel ischemia. Note the two adjacent collapsed segments of small bowel (arrows), where the loop of small bowel had entered an internal hernia tion, with collapse of the colon. The small bowel may demonstrate varying degrees of associated dilation. Colonic volvulus should be readily identified (Fig. 13.11), particularly on review of multi-planar reformations. A variety of CT signs have been reported, and volvulus may be complicated by ischemia and subsequent perforation. Marked dilation of most or all of the colon may be seen in toxic megacolon from a variety of etiologies, as well as in "pseudo-obstruction," the latter of which also can lead to subsequent volvulus. A very common cause of abdominal pain in emergency department patients is constipation, which may be of varying degrees of severity, usually without an associated focal obstructing lesion. Other motility disorders, such as scleroderma and
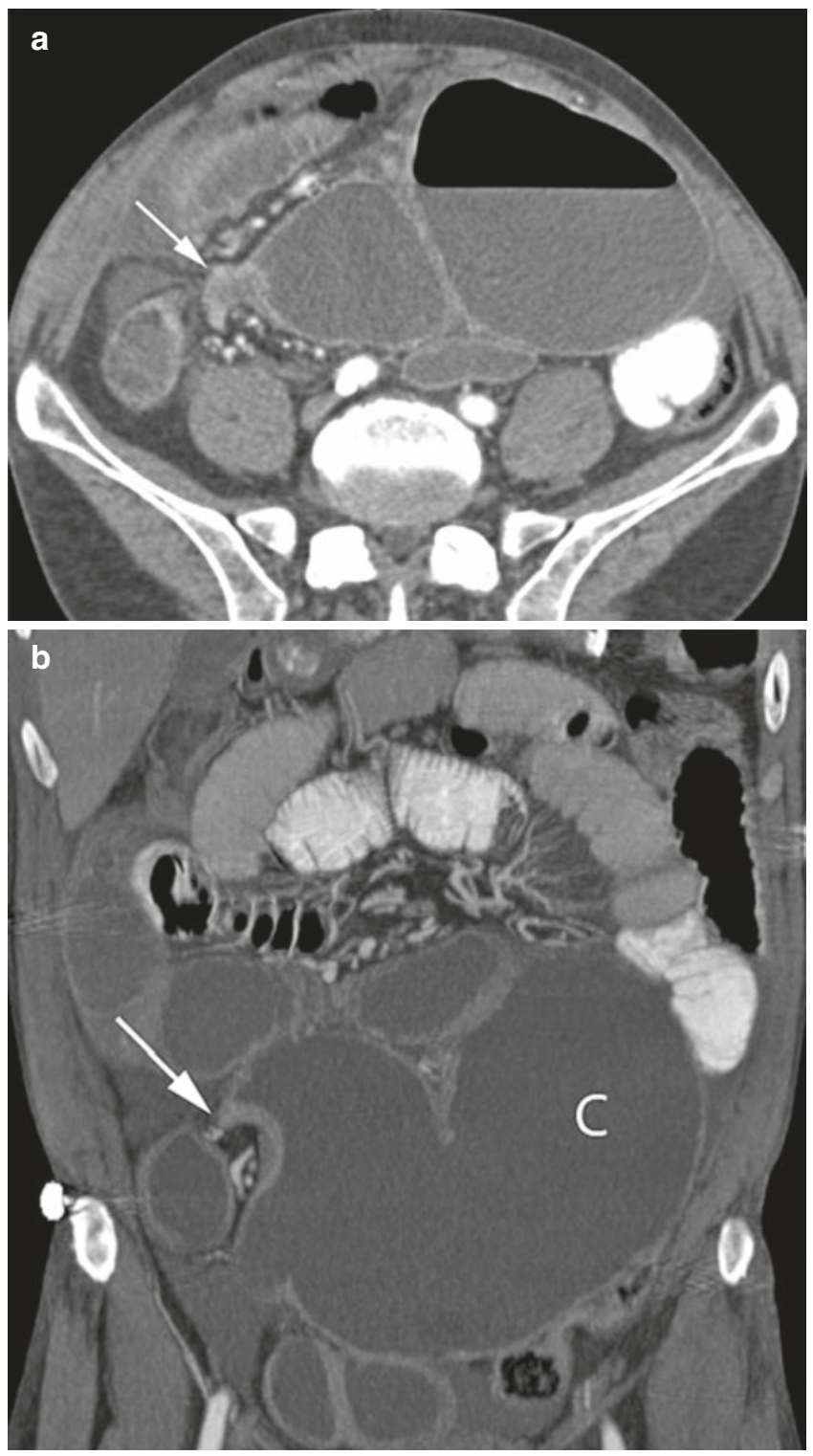

Fig.13.11 (a, b) Cecal volvulus. Transverse CT image (a) and coronal CT reformation (b) demonstrate a markedly dilated cecum $(C)$ located in the midline and left lower abdomen and upper pelvis. The arrow points to the area of colonic twisting. Note the dilated small bowel loops, due to the proximal colonic obstruction 

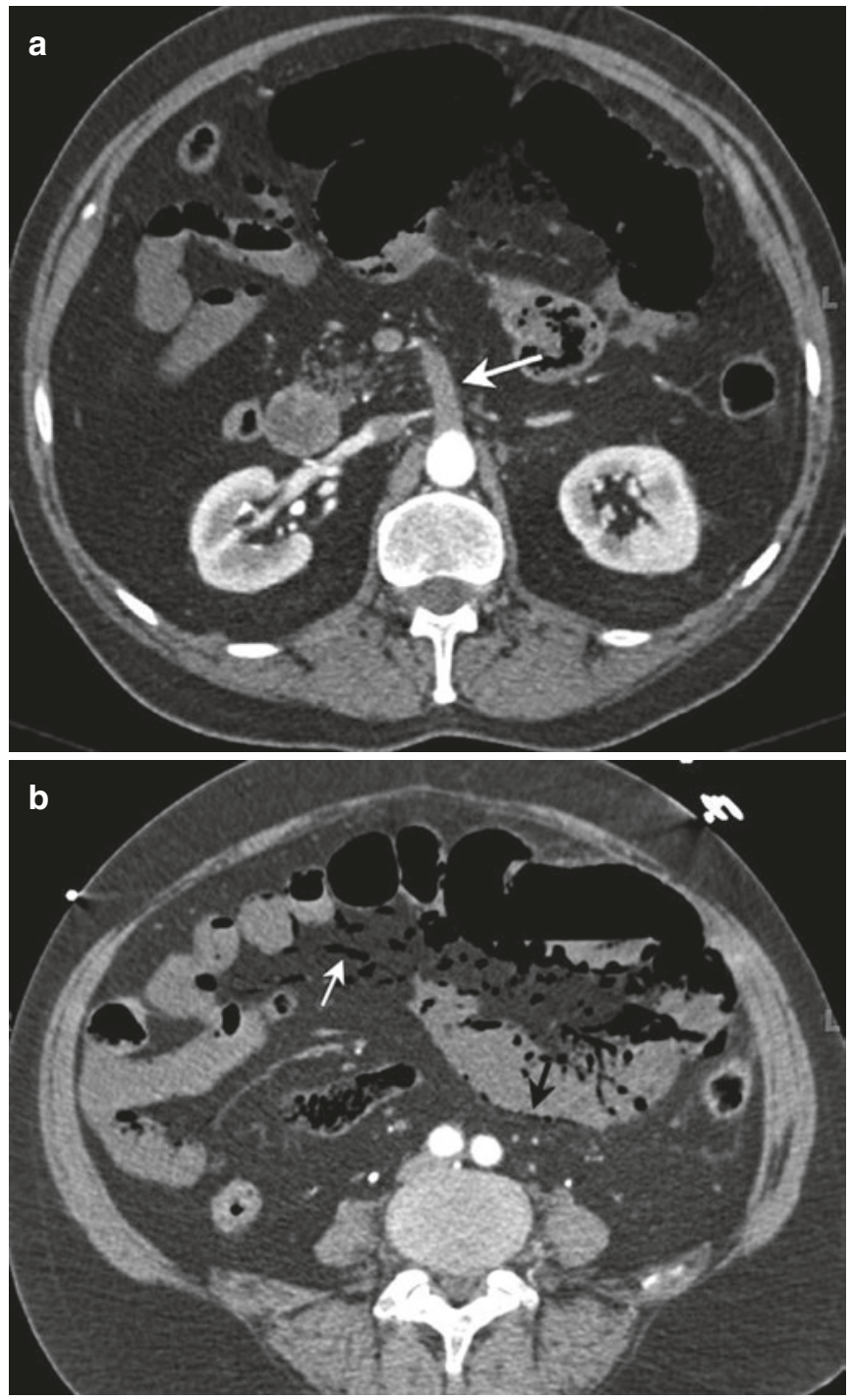

Fig. 13.12 Acute bowel ischemia secondary to superior mesenteric artery thrombosis. 52-year-old man with 4-day history of severe diffuse abdominal pain. Transaxial (a, b) and sagittal curved planar reformatted (c) contrast-enhanced CT images demonstrate thrombosis of the

administration of anticholinergic or opioid medications, also may lead to the performance of $\mathrm{CT}$, in patients presenting with acute abdominal pain.

One of the most important responsibilities of the radiologist in the case of acute bowel obstruction is to help determine if the patient should undergo surgery or conservative management. Today, more than $50 \%$ of patients with bowel obstruction do not undergo surgery. Imaging criteria for surgical intervention include findings indicative of bowel ischemia (see Sect. 13.4.2) or a closed-loop obstruction.

\subsubsection{Bowel Ischemia}

Bowel ischemia has a variety of etiologies, including arterial thrombosis or embolism, venous thrombosis, hypoperfusion, and vasculitis. Occlusive disease involves the mesenteric arter-

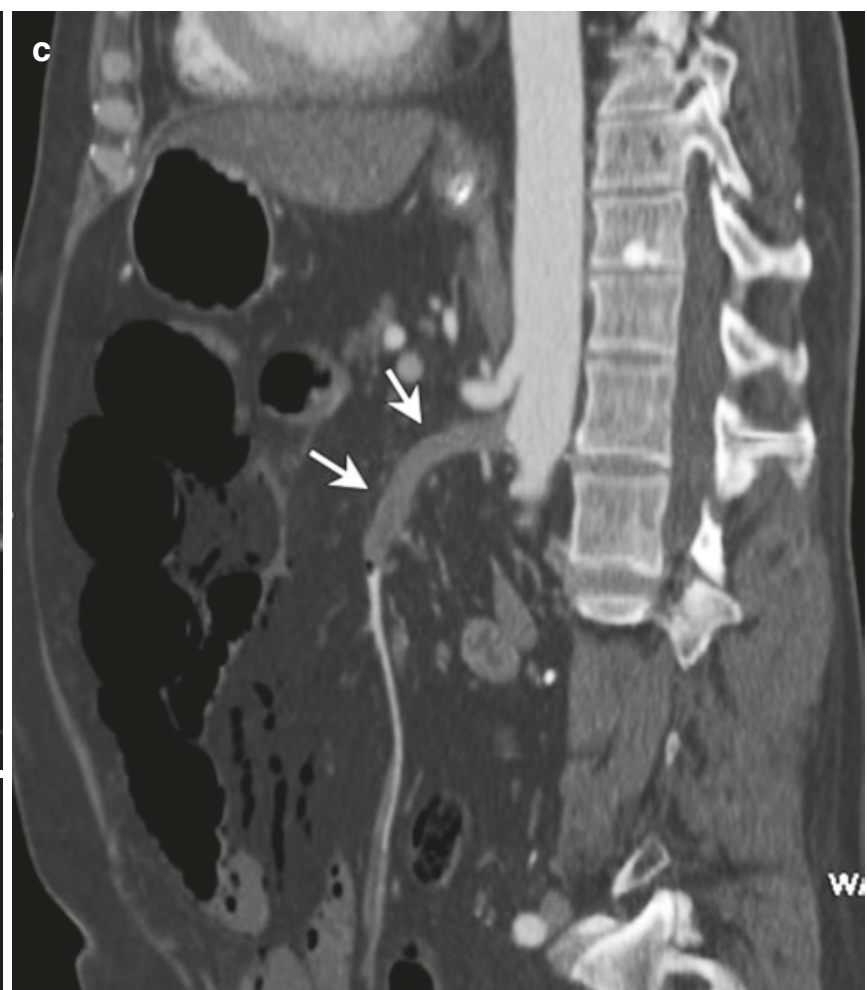

superior mesenteric artery (large white arrow). Note the bowel pneumatosis (small black arrow) and gas within the mesenteric veins (small white arrow) indicative of bowel ischemia

ies, most commonly the superior mesenteric artery (Fig. 13.12), whereas bowel ischemia secondary to venous thrombosis is much less common. The only direct CT (or MRI) sign of vascular impairment of the bowel is diminished wall enhancement, whereas increased bowel wall enhancement is seen in a wide variety of disorders, including in a subset of patients with bowel ischemia due to reactive hyperemia, loss of autoregulation, or compromised venous outflow. Other cross-sectional imaging findings include direct visualization of embolus or thrombus in the mesenteric arterial circulation or thrombus in the mesenteric venous circulation. Bowel distension and wall edema are non-specific findings that may be identified in ischemia, but also may be present in various infectious, inflammatory, or immunologic conditions and in primary bowel obstruction. Nonocclusive acute mesenteric ischemia usually is due to hypoperfusion secondary to severe cardiac disease; generalized decreased caliber of the arterial circulation is present. 


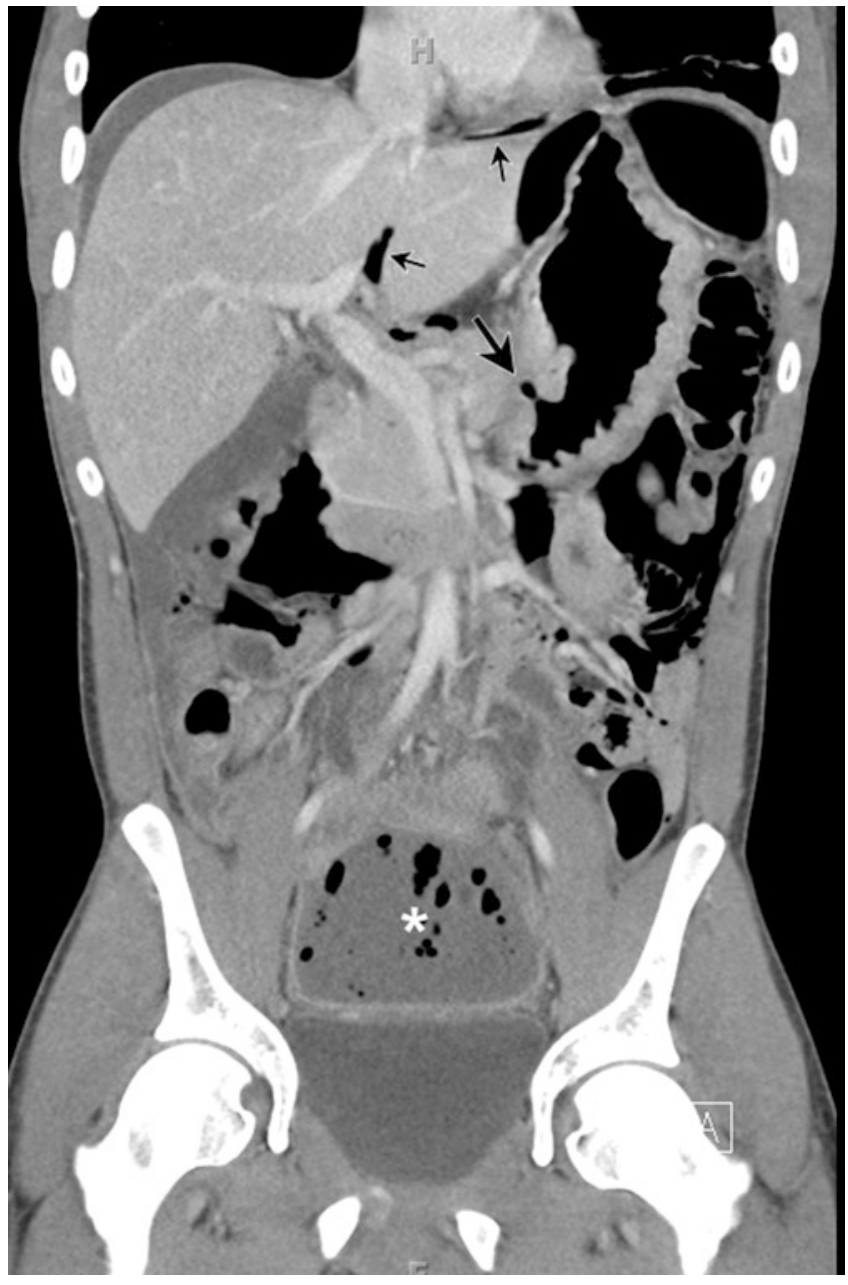

Fig. 13.13 Perforated gastric ulcer. 26-year-old man with left upper quadrant pain, fever, and leukocytosis. Coronal CT image shows an ulcer (large arrow) along the lesser curvature of the stomach with surrounding wall thickening. Note free intraperitoneal gas (small arrows) and large pelvic abscess (asterisk)

Bowel ischemia also may be secondary to bowel obstruction, particularly a closed-loop SBO, but may also be identified secondary to large bowel obstruction or volvulus. Focal or regional bowel wall thickening due to edema and hemorrhage may be seen on CT, with decreased bowel wall enhancement and edema adjacent to the affected bowel. With frank infarction, pneumatosis, portal/mesenteric venous gas, peritonitis/ascites, and free gas can be found.

\subsubsection{Gastrointestinal Perforation}

Gastrointestinal perforation usually causes localized pain initially, which becomes more diffuse if peritonitis develops. Perforation due to peptic ulcer disease (Fig. 13.13) or a necrotic neoplasm has become less frequent, due to earlier diagnosis and improved therapy (Fig. 13.12a). However, perforation resulting from endoscopic procedures has increased in incidence. Perforation of the small bowel is relatively uncommon, but may be secondary to a foreign body, diverticulitis, or trauma, among other possibilities. Perforation of the colon is more frequent and can occur when the colon becomes markedly distended proximal to an obstructing lesion (e.g., tumor, volvulus) or when the colonic wall is friable (e.g., diverticulitis, ischemia, ulcerative colitis, tumor).

Pneumoperitoneum can be recognized by gas under the diaphragm on an upright chest radiograph or an upright or lateral decubitus abdominal radiograph. Detection of subtle pneumoperitoneum often is difficult. $\mathrm{CT}$ is the examination of choice in suspected bowel perforation as is much more sensitive for the identification of such perforations compared with plain radiographs and can be used to identify the source of perforation in the vast majority of such cases. Viewing the CT images using lung window settings improves demonstration of small amounts of extraluminal gas. Examining all three orthogonal planes (axial, sagittal, and coronal) improves the sensitivity for detection of small volume pneumoperitoneum. The sagittal plane is especially helpful as a minimal amount of gas in the umbilical area may be very difficult to see under the transverse mesocolon on axial images.

Identifying the cause of perforation may be difficult. The first step is to determine if the perforation is in the upper or lower abdomen. Some simple rules are helpful. If the pneumoperitoneum is localized only under the transverse mesocolon, the perforation likely is of colonic origin. If gas accumulates in the lesser omentum and along the left portal vein in the liver hilum, gastroduodenal perforation is likely. If gas is found in the lesser sac, gastroduodenal perforation is almost certain. In cases of abundant pneumoperitoneum, localizing the site of perforation may be difficult.

Gas from retroperitoneal bowel perforation typically has a more mottled appearance and initially may be contained locally. As with intraperitoneal perforation, it is more readily identified and its etiology more clearly defined on CT. Diagnostic considerations include a perforated duodenal ulcer, complicated diverticulitis, perforated colon cancer, and iatrogenic perforation.

\subsubsection{Acute Abdomen with Flank or Epigastric Pain}

Acute flank pain or upper abdominal pain that radiates to the back most commonly is a manifestation of retroperitoneal pathology, particularly ureteral colic, acute pancreatitis, and ruptured abdominal aortic aneurysm (AAA). If AAA rupture is suspected, or if retroperitoneal (or other site of hemorrhage) is suspected, then an emergency non-contrast CT is performed. An additional CT acquisition with IV contrast to evaluate the vascular anatomy in more detail and to determine if there is ongoing active hemorrhage usually is obtained as part of the same examination. 

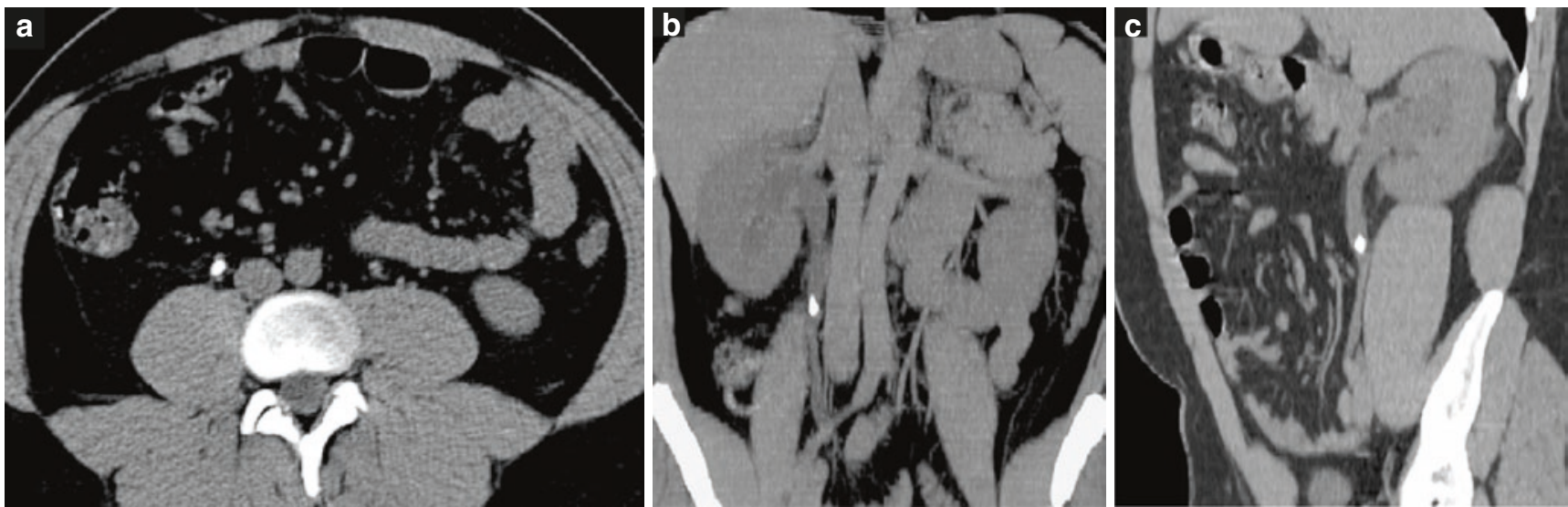

Fig. 13.14 (a-c) Right-sided urinary colic. Axial CT image (a) and coronal (b) and oblique sagittal (c) CT reformations show dilatation of the proximal right urinary tract, due to a ureteral calculus

\subsubsection{Urinary Colic}

The lifetime incidence of an obstructing ureteral calculus is estimated at upward of $12 \%$. The clinical diagnosis often is not initially clear (and CT with IV contrast may therefore be obtained). For evaluation of known or suspected urolithiasis, non-contrast $\mathrm{CT}$ is the imaging examination of choice. It is extremely accurate for the identification of urinary tract calculi, as well as for stone sizing, determination of overall stone burden, identification of complications, and demonstration of alternative or additional significant diagnoses (in upward of $10-15 \%$ of cases).

Virtually all urinary tract stones are radiopaque, regardless of their specific chemical composition. The non-symptomatic side acts as an intrinsic control, as bilateral ureteral calculi are rare. Secondary signs of obstruction, including hydronephrosis, hydroureter, renal swelling, and perinephric and periureteric edema, are present in $95 \%$ of patients with a ureteral stone (Fig. 13.14). Coronal reformations are routinely used and may help the radiologist distinguish between a ureteral calculus and an adjacent phlebolith. Phleboliths, which are calcifications in veins, increase in incidence with age, usually are located lateral and posterior to the ureter, and typically have a "tail" or "comet" sign, whereas most ureteral calculi are surrounded by a rim of soft tissue. In patients who present with symptoms of renal colic but do not have a ureteral calculus, CT demonstrates a wide variety of alternative diagnoses in a substantial minority of such patients.

$\mathrm{CT}$ is used to determine management of patients with urolithiasis, as almost all ureteral calculi $<5 \mathrm{~mm}$, especially if distally located, will pass spontaneously with conservative treatment (unless there is concurrent infection, in which case more aggressive management usually is indicated). Renal calculi frequently are found concurrently with ureteral calculi and occasionally may be the source of pain. Repeat CT, for short-term follow-up and for additional episodes of colic, should be used selectively, especially in younger patients, and attention should be paid to reducing radiation dose. In very selected situations, repeat CT with IV contrast is indicated, for evaluation of suspected superinfection or to clarify an equivocal alternative diagnosis.

\subsubsection{Acute Pancreatitis}

Pancreatitis is common and has a broad spectrum of presentations and outcomes. On initial clinical evaluation, pancreatitis frequently is confused with other disorders, and amylase and lipase levels may either be pending or not obtained. Therefore it is important for the radiologist to identify pancreatitis, usually on CT but occasionally on sonography, when the diagnosis is not suspected clinically. The vast majority of cases are due to gallstones or alcohol abuse. Less common etiologies include trauma, interventional pancreatobiliary procedures, medications, elevated triglycerides, congenital anomalies (i.e., pancreas divisum, annular pancreas, absent dorsal pancreas), and underlying tumor. Less common presentations include chronic pancreatitis, autoimmune pancreatitis, and paraduodenal (groove) pancreatitis.

A routine CT protocol is used when pancreatitis is not specifically suspected, but a tailored protocol can be used when it is suspected or for follow-up of known pancreatitis. Specifically, no oral contrast is given, or water is used as a neutral oral contrast agent. IV contrast is administered, if not contraindicated, to assess for necrosis, to characterize fluid collections, and to identify vascular complications including venous thrombosis and pseudoaneurysm formation. Imaging is performed in a late arterial ("pancreatic") phase. CT findings range from a normal or nearly normal pancreas with mild focal or diffuse peripancreatic inflammation to marked pancreatic edema and associated necrosis (Fig. 13.15). In the initial few days, CT findings do not necessarily correlate with patient outcome, but later in the patient's course, the CT findings have greater prognostic significance. 

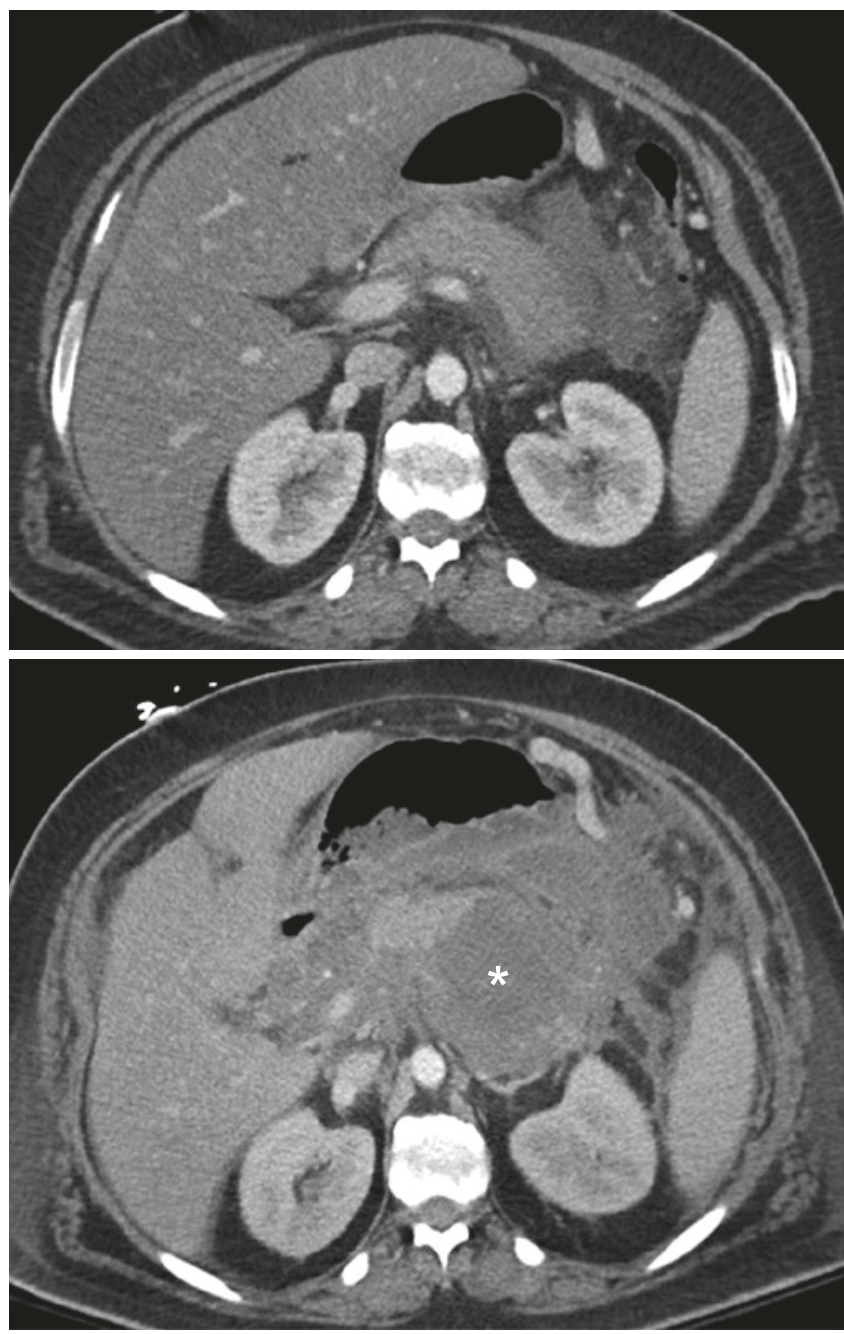

Fig.13.15 Evolution of acute interstitial edematous pancreatitis (a) to acute necrotizing pancreatitis (b). 34-year-old man with abdominal pain. (a) Transaxial contrast-enhanced CT image shows diffuse edema of the pancreas and peripancreatic fluid. (b) Transaxial contrast-enhanced CT image obtained 2 weeks later shows necrosis of the pancreatic tail which is now replaced by an acute necrotic collection (asterisk)

There are two main types of acute pancreatitis: interstitial edematous versus necrotizing. The revised Atlanta classification system attempts to better characterize the disease process, to standardize terminology-including the description of cross-sectional imaging findings-and to provide better correlation with prognosis. Other CT scoring systems also are in use to characterize the findings of pancreatitis and provide prognostic information. In the revised Atlanta classification system, in the first 4 weeks, there may be acute peripancreatic fluid collections (APFCs) associated with interstitial edematous pancreatitis versus acute necrotic collections (ANCs) associated with necrotizing pancreatitis, the latter of which can be pancreatic and/or peripancreatic and which may be sterile or infected. After 4 weeks, APFCs usually resolve, but can become pseudocysts (which may be sterile or infected), whereas ANCs may evolve into areas of walled-off necrosis
(WON), which may be pancreatic and/or peripancreatic and which may be sterile or infected.

CT-guided aspiration is very useful when indicated, to distinguish sterile from infected necrosis/fluid collections. Additionally, MRI should be strongly considered when followup imaging is needed, to reduce radiation exposure, as these patients often require multiple repeat examinations in the short term and may also have repetitive episodes of pancreatitis.

\subsection{Imaging of Abdominal and Pelvic Trauma}

\subsubsection{Blunt Abdominal Trauma}

MDCT has become the primary imaging examination for evaluating blunt abdominal trauma and has replaced diagnostic peritoneal lavage (DPL) in almost all circumstances. US plays an important role in the detection of hemoperitoneum in hemodynamically unstable patients. The intra-abdominal injuries expected after blunt abdominal trauma depend upon the mechanism of injury, whether or not a restraint device was used, and patient body habitus. Knowledge of the common patterns of injury resulting from different types of motor vehicle collisions (frontal, lateral, and rear impact; sideswipe, rollover, etc.) and other traumatic events aids identification of specific types of injuries, but a discussion of these factors is beyond the scope of this chapter.

\subsubsection{Imaging Techniques}

Intravenous contrast enhancement for MDCT is critical to enable detection of abdominal and pelvic injuries. As noted above, mages generally are obtained in the portal venous phase of enhancement (approximately 65-80 s after administration of $100-150 \mathrm{~mL}$ injected at $3-5 \mathrm{~mL}$ per second). Arterial phase images may be helpful in some severely injured patients to demonstrate vascular injuries of the solid organs that may not be apparent on the portal venous phase images and to characterize areas of active extravasation as arterial versus venous. When injuries are suspected on the portal venous phase images, 5-10-min delayed images are helpful to detect injuries of the urinary tract and to further characterize solid visceral organ injuries that involve the vasculature. Patients with suspected bladder injury should undergo CT cystography. Oral contrast material is no longer administered at almost all trauma centers for patients with blunt abdominal trauma.

Focused assessment with sonography for trauma (FAST) is a limited abdominal ultrasound examination designed primarily to identify peritoneal fluid. A six-point examination generally is performed using a $3.5 \mathrm{MHz}$ sector transducer. Images of the right subphrenic space (above the 
liver), left subphrenic space (above the spleen), hepatorenal fossa, perisplenic space (at the inferior margin of the spleen), peritoneal recess of the pelvis, and pericardium are recorded. Although FAST has been shown to be $86 \%$ sensitive for detection of abdominal injuries requiring surgery, liver and spleen injuries are not always associated with hemoperitoneum, and a negative FAST does not exclude an intra-abdominal injury. On the other hand, patients with a positive FAST do not always require surgery. Therefore, hemodynamically stable patients with a positive FAST should undergo MDCT for staging of their injuries. In addition, FAST is not reliable for assessment of the retroperitoneum.

\subsubsection{Pneumoperitoneum}

On MDCT, pneumoperitoneum may consist of free gas beneath the anterior abdominal wall or small bubbles of gas trapped between leaves of the mesentery or within peritoneal recesses. Review of images using lung window settings can be helpful in identifying small amounts of intraperitoneal gas. In the setting of trauma, pneumoperitoneum is highly suggestive of bowel perforation. A localized collection of gas may be identified adjacent to the site of bowel perforation. It is important to keep in mind that pneumoperitoneum is not always present on CT scans of patients with documented bowel perforation. In addition, pneumoperitoneum occasionally may be present in patients without bowel injury, secondary to multiple other potential causes including barotrauma, intraperitoneal spread of air from a large pneumothorax, or DLP.

\subsubsection{Peritoneal Fluid}

In trauma patients, peritoneal fluid most commonly represents blood resulting from injury to the liver, spleen, bowel, or mesentery. In most patients hemoperitoneum has an attenuation value ranging from 20 to $45 \mathrm{HU}$ or greater (Fig. 13.16); however, in up to one-quarter of patients, hemoperitoneum has an attenuation value less than $20 \mathrm{HU}$. Blood located adjacent to the source of hemorrhage often is partially clotted and higher in attenuation (the "sentinel clot" sign), which can be helpful in identifying the site of bleeding. Free peritoneal fluid in the absence of direct signs of solid or hollow visceral injury may be problematic, especially in men. However, recent studies have shown that small amounts of simple peritoneal fluid are found in up to $5 \%$ of male blunt trauma patients in the absence of hollow or solid organ injury. Therefore, immediate surgical intervention is not mandated in such patients, but careful review of the CT images for subtle injuries and hospital admission with close clinical follow-up and repeat $\mathrm{CT}$ if necessary are recommended.
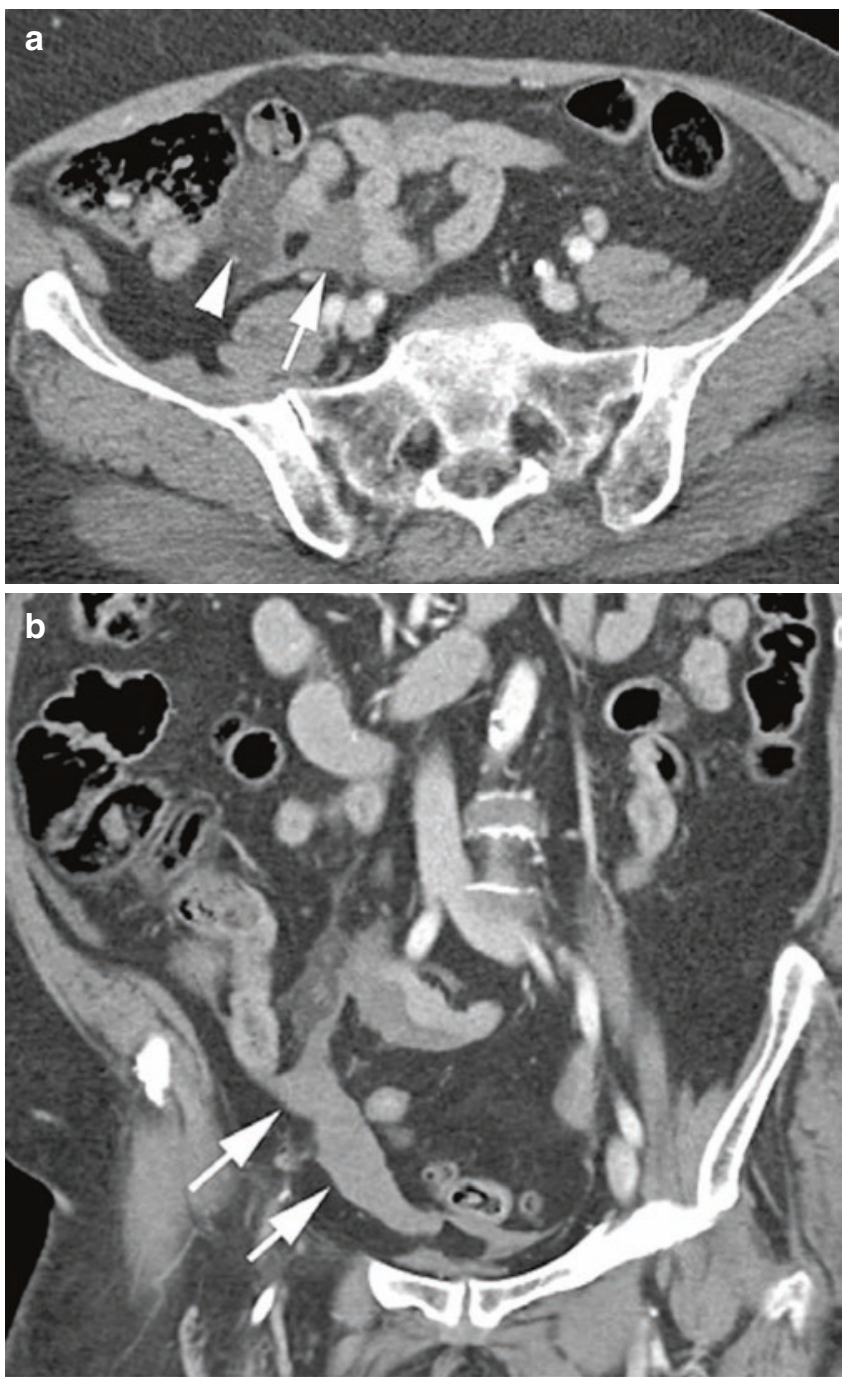

Fig. 13.16 (a, b). Hemoperitoneum due to mesenteric injury from motor vehicle collision. Transaxial CT image (a) demonstrates highattenuation fluid (arrow) adjacent to small bowel loops. Increased attenuation within the mesenteric fat (arrowhead) is due to a mesenteric contusion. Coronal image (b) shows blood layering dependently within the peritoneal cavity

\subsubsection{Splenic Injuries}

The spleen is the most commonly injured abdominal organ in blunt trauma. In the large majority of cases, splenic injuries can be managed nonsurgically. The need for surgical intervention is based on patient factors, clinical findings, and the CT-based splenic injury scale developed by the American Association for the Surgery of Trauma (AAST) (Table 13.1). The AAST splenic injury scale takes into account the size and location of splenic lacerations and hematomas. Higher-grade injuries (generally AAST grade III and higher) more often require surgical therapy; however, this grading scale has been shown to be a relatively poor predictor of the success of nonsurgical management. More recently an MDCT-based splenic injury grading sys- 
Table 13.1 American Association for the Surgery of Trauma (AAST) Splenic Injury Scale

\begin{tabular}{|c|c|}
\hline $\begin{array}{l}\text { Injury grade and } \\
\text { type }\end{array}$ & Description \\
\hline \multicolumn{2}{|l|}{$I$} \\
\hline Hematoma & Subcapsular, $<10 \%$ surface area \\
\hline Laceration & Capsular tear, $<1-\mathrm{cm}$ parenchymal depth \\
\hline \multicolumn{2}{|r|}{ 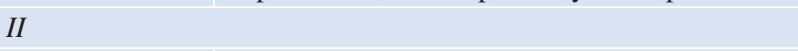 } \\
\hline Hematoma & $\begin{array}{l}\text { Subcapsular, } 10-50 \% \text { of surface area or } \\
\text { intraparenchymal hematoma }<5-\mathrm{cm} \text { diameter }\end{array}$ \\
\hline Laceration & $\begin{array}{l}1-3 \mathrm{~cm} \text { parenchymal depth; does not involve } \\
\text { trabecular vessels }\end{array}$ \\
\hline \multicolumn{2}{|l|}{ III } \\
\hline Hematoma & $\begin{array}{l}\text { Subcapsular hematoma, }>50 \% \text { surface area or } \\
\text { expanding; ruptured subcapsular or } \\
\text { intraparenchymal hematoma; intraparenchymal } \\
\text { hematoma }>5 \mathrm{~cm} \text { or expanding }\end{array}$ \\
\hline Laceration & $\begin{array}{l}>3-\mathrm{cm} \text { parenchymal depth or involves trabecular } \\
\text { vessels }\end{array}$ \\
\hline \multicolumn{2}{|r|}{ 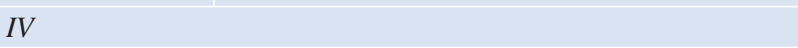 } \\
\hline Laceration & $\begin{array}{l}\text { Laceration, involves segmental or hilar vessels, } \\
\text { producing major devascularization ( }>25 \% \text { of } \\
\text { spleen) }\end{array}$ \\
\hline \multicolumn{2}{|r|}{ 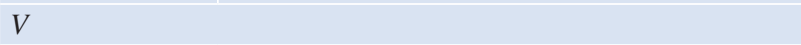 } \\
\hline Laceration & Completely shattered spleen \\
\hline Vascular & Hilar vascular injury that devascularizes spleen \\
\hline
\end{tabular}

Note: Increase by one grade (up to grade III) for multiple injuries. Adapted from Moore et al. (1995), with permission

Table 13.2 Multidetector computed-tomography-based splenic injury grading system

\begin{tabular}{|c|c|}
\hline $\begin{array}{l}\text { Injury } \\
\text { grade }\end{array}$ & Description \\
\hline I & $\begin{array}{l}\text { Subcapsular hematoma }<1-\mathrm{cm} \text { thick; laceration }<1-\mathrm{cm} \\
\text { parenchymal depth; parenchymal hematoma }<1-\mathrm{cm} \\
\text { diameter }\end{array}$ \\
\hline II & $\begin{array}{l}\text { Subcapsular hematoma } 1 \text { - to } 3 \text {-cm thick; laceration } 1 \text { - to } \\
\text { 3-cm parenchymal depth; parenchymal hematoma } 1 \text { - to } \\
\text { 3-cm diameter }\end{array}$ \\
\hline III & $\begin{array}{l}\text { Splenic capsular disruption; subcapsular hematoma }<3-\mathrm{cm} \\
\text { thick; laceration }<3-\mathrm{cm} \text { parenchymal depth; parenchymal } \\
\text { hematoma }<3-\mathrm{cm} \text { thick }\end{array}$ \\
\hline IVA & $\begin{array}{l}\text { Active intraparenchymal or subcapsular splenic bleeding; } \\
\text { splenic vascular injury (pseudoaneurysm or arteriovenous } \\
\text { fistula); shattered spleen }\end{array}$ \\
\hline IVB & Active intraperitoneal bleeding \\
\hline
\end{tabular}

Adapted from Marmery et al. (2007), with permission

tem (Table 13.2) has been proposed, which takes into account additional CT features of splenic trauma including the presence of active hemorrhage (Fig. 13.17), a pseudoaneurysm, or an arteriovenous (AV) fistula. Active hemorrhage appears as a localized area of hyperattenuation within the injured splenic parenchyma, which persists and grows larger on delayed images. In contradistinction, a pseudoan-
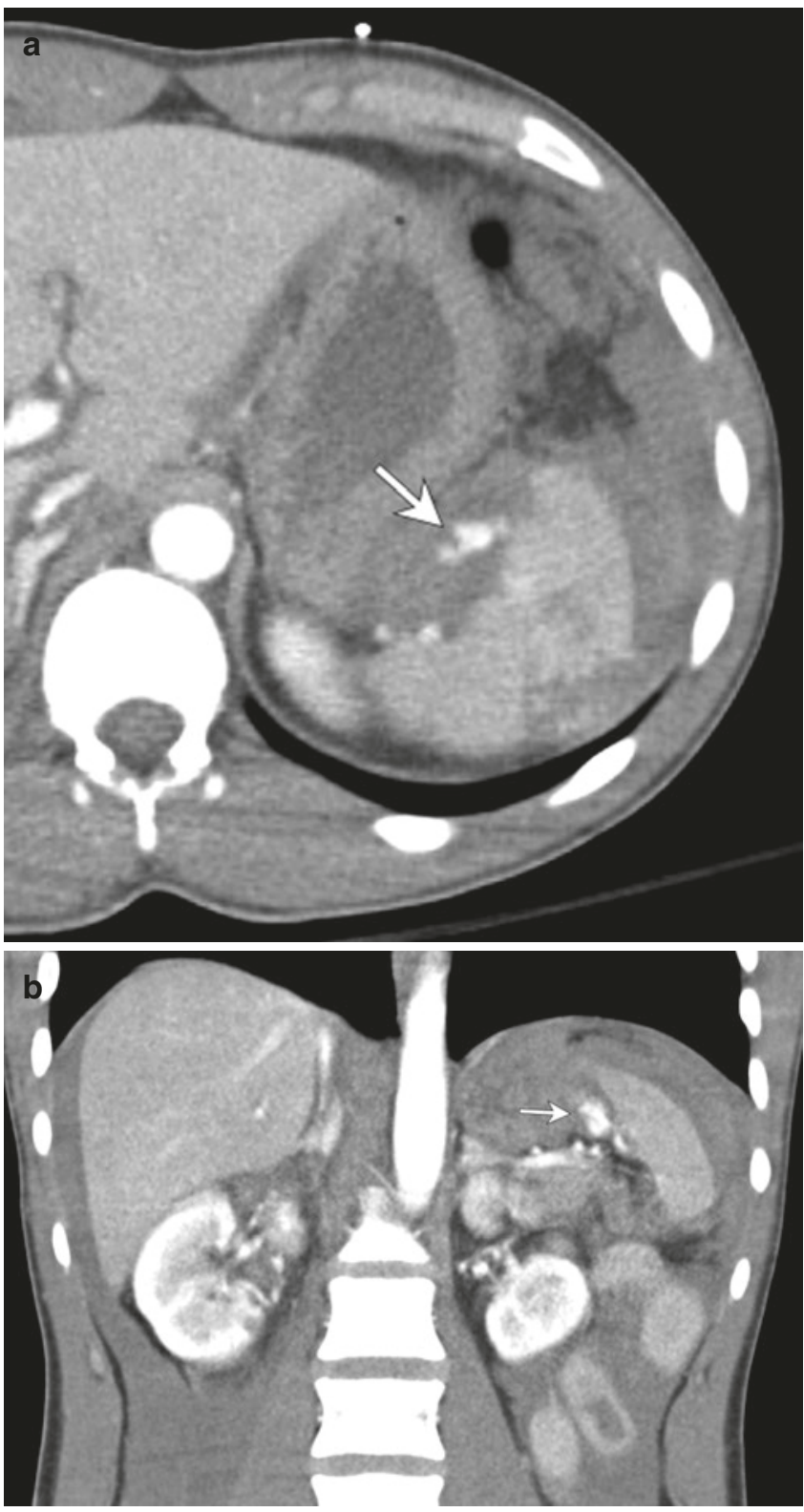

Fig. 13.17 Splenic laceration. Transaxial (a) and coronal (b) contrastenhanced CT images show a splenic laceration with contrast extravasation (arrow) and intraperitoneal bleeding (grade IVB)

eurysm or AV fistula washes out on delayed phase images. Some trauma specialists now advocate the acquisition of arterial phase CT images of the abdomen, in addition to the portal venous and delayed phase images after blunt trauma, because not all vascular injuries of the spleen are identified on portal venous and delayed phase images. Further study is required to validate this proposal.

\subsubsection{Hepatic Injuries}

Hepatic injuries are only slightly less common than splenic injuries and more frequently involve the right lobe. As with 
Table 13.3 American Association for the Surgery of Trauma (AAST) Liver Injury Scale

\begin{tabular}{|c|c|}
\hline $\begin{array}{l}\text { Injury grade } \\
\text { and type }\end{array}$ & Description \\
\hline \multicolumn{2}{|l|}{1} \\
\hline Hematoma & Subcapsular, $<10 \%$ surface area \\
\hline Laceration & $<1 \mathrm{~cm}$ in depth \\
\hline \multicolumn{2}{|l|}{2} \\
\hline Hematoma & $\begin{array}{l}\text { Subcapsular, } 10-50 \% \text { of surface area; } \\
\text { intraparenchymal hematoma }<10 \mathrm{~cm} \text { in diameter }\end{array}$ \\
\hline Laceration & $1-3 \mathrm{~cm}$ in depth or $<10 \mathrm{~cm}$ in length \\
\hline \multicolumn{2}{|r|}{ 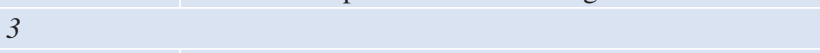 } \\
\hline Hematoma & $\begin{array}{l}\text { Subcapsular, }>50 \% \text { surface area or expanding; } \\
\text { ruptured subcapsular or parenchymal hematoma; } \\
\text { intraparenchymal hematoma }>10 \mathrm{~cm} \text { or expanding }\end{array}$ \\
\hline Laceration & $>3$-cm parenchymal depth \\
\hline \multicolumn{2}{|l|}{4} \\
\hline Laceration & $\begin{array}{l}\text { Parenchymal disruption involving } 25-75 \% \text { of hepatic } \\
\text { lobe or one to three Couinaud segments in a single } \\
\text { lobe }\end{array}$ \\
\hline \multicolumn{2}{|r|}{ 党 } \\
\hline Laceration & $\begin{array}{l}\text { Parenchymal disruption involving }>75 \% \text { of hepatic } \\
\text { lobe, or more than three Couinaud segments in a } \\
\text { single lobe }\end{array}$ \\
\hline Vascular & $\begin{array}{l}\text { Juxtahepatic venous injuries (i.e., retrohepatic vena } \\
\text { cava and/or central major hepatic veins) }\end{array}$ \\
\hline \multicolumn{2}{|l|}{6} \\
\hline Vascular & Hepatic avulsion \\
\hline
\end{tabular}

Adapted from Moore et al. (1995), with permission

splenic injuries, the majority of blunt liver injuries are successfully managed nonoperatively. The AAST liver injury scale (Table 13.3) is commonly used to assess the severity of hepatic injuries. Lacerations appear as linear or branching areas of hypoattenuation that frequently travel along vascular planes. Perihilar lacerations may be associated with biliary tract injuries. As with the AAST splenic injury scale, the AAST liver injury scale has limitations in guiding clinical management. Additional useful CT findings include extension of the injury to the major hepatic veins (Fig. 13.18), the presence of active bleeding into the peritoneal cavity, and the presence of large volume hemoperitoneum. Extension to the major hepatic veins usually requires surgery to control hemorrhage, and active bleeding into the peritoneum often can be treated with endovascular interventions.

\subsubsection{Bowel and Mesenteric Injuries}

Injuries to the hollow viscera and mesentery occur in only approximately $5 \%$ of patients with blunt abdominal trauma, and the CT findings may be subtle; however, identification of bowel and mesenteric injuries is critical, as delay in diagnosis increases the morbidity and mortality from peritonitis and sepsis. The bowel segments affected most commonly are the proximal jejunum and the distal ileum. The most specific signs of bowel

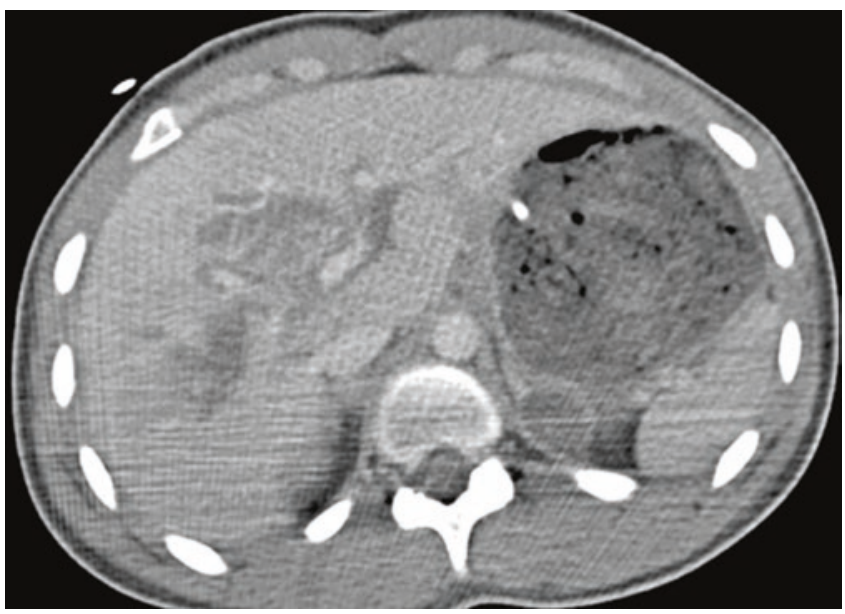

Fig. 13.18 Grade V liver laceration. Transaxial CT image shows a large ill-defined area of hypoattenuation surrounding the central hepatic blood vessels

injury are focal discontinuity of the bowel wall (transection), extraluminal oral contrast (when administered), pneumoperitoneum, and retropneumoperitoneum. Less specific (but more frequently encountered) signs of bowel trauma include focal wall thickening, abnormal bowel wall enhancement, mesenteric stranding, and free intraperitoneal fluid. The association of a focal bowel abnormality with adjacent or free fluid increases the likelihood that bowel injury is present. The most specific signs of mesenteric injury are active extravasation from a mesenteric vessel (Fig. 13.19), mesenteric hematoma, and abrupt termination or beading of mesenteric vessels. Small isolated mesenteric hematomas do not always require immediate surgery and can be managed with observation. Larger hematomas and mesenteric vascular injuries have a higher risk of subsequent bowel ischemia and usually require surgical repair.

In the setting of trauma, the combination of diffuse thickening and hyperenhancement of the bowel generally is not an indication of direct bowel injury but is a component of the hypoperfusion complex (Fig. 13.20), which is seen in trauma patients who have persistent hypovolemia after initial intravenous fluid resuscitation. Other $\mathrm{CT}$ features of the hypoperfusion complex include collapse of the inferior vena cava, flattening of the renal veins, decreased caliber of the aorta, delayed nephrograms, increased enhancement of the adrenal glands, decreased enhancement of the spleen, and pancreatic enlargement with peripancreatic and retroperitoneal edema. These findings usually are reversible with additional fluid resuscitation.

\subsubsection{Pancreatic Injuries}

Pancreatic injuries typically occur after blunt trauma to the upper abdomen. Pancreatic contusions appear as focal areas of hypoattenuation or enlargement, whereas lacerations are linear low attenuation defects that may be superficial or 

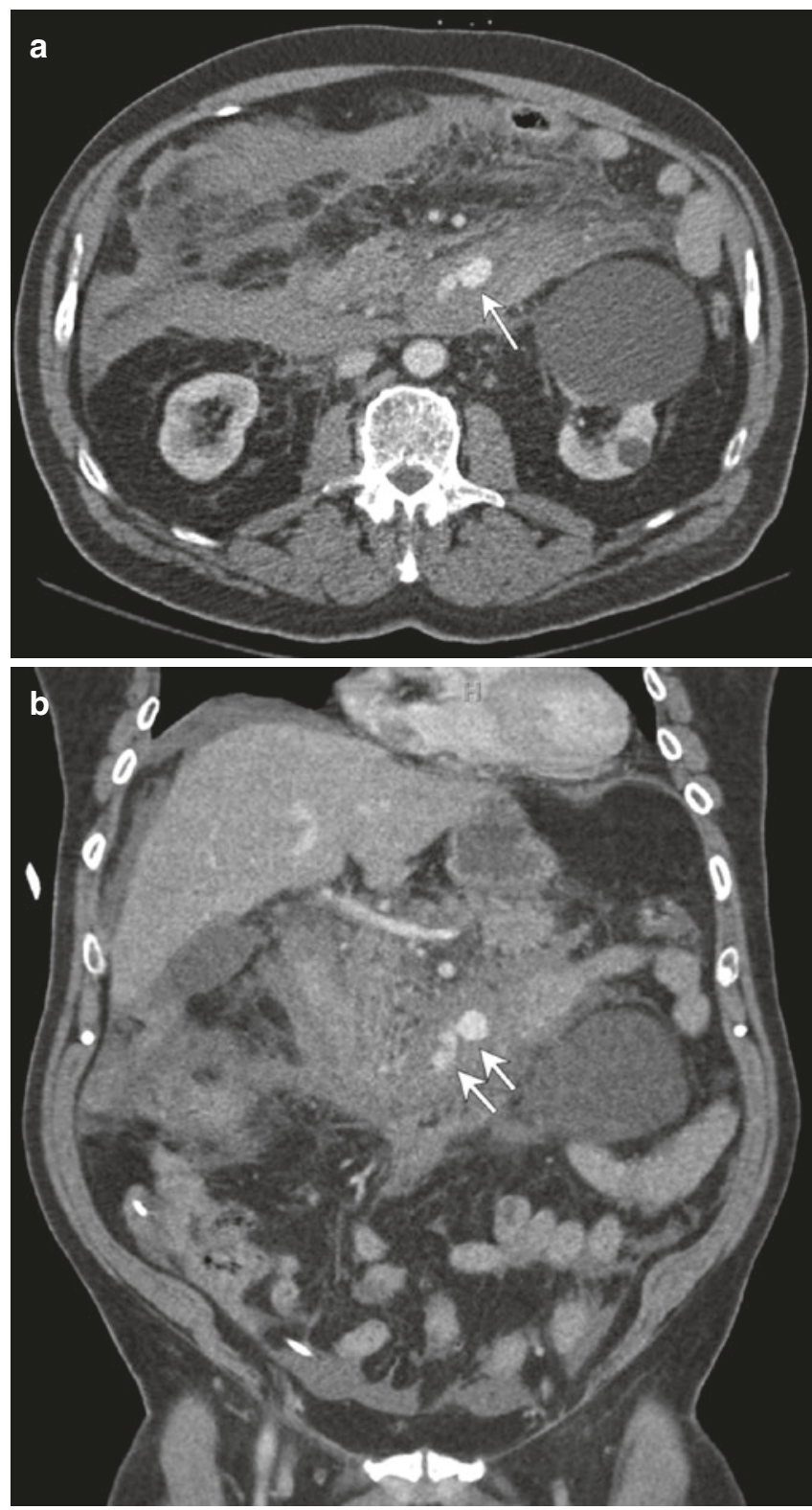

Fig. 13.19 Severe mesenteric injury. 81-year-old man who suffered blunt abdominal trauma from a fall. Transaxial (a) and coronal (b) contrast-enhanced CT images demonstrate a large mesenteric hematoma with extravasation from a mesenteric artery (arrows)

extend completely through the pancreas, resulting in transection (Fig. 13.21). Lacerations that involve more than $50 \%$ of the pancreatic thickness usually cause pancreatic ductal injury. Indirect signs of pancreatic injury include fluid in the peripancreatic fat or transverse mesocolon and thickening of the left anterior renal fascia. The injured pancreas may appear normal at CT in the first 12 hours after trauma. Therefore a $24-48-\mathrm{h}$ repeat CT is warranted in patients who subsequently develop abdominal pain.

Magnetic resonance cholangiopancreatography (MRCP) may be useful to document noninvasively injury of the main pancreatic duct; however, endoscopic retrograde cholangiopancreatography (ERCP) may be necessary for diagnosis. Although some patients with pancreatic ductal injury may be successfully treated with endoscopic pancreatic duct stenting, most require surgical repair.

\subsubsection{Urinary Tract Injuries}

As with the liver and spleen, an AAST grading system classifies the severity of renal trauma based on the size and location of renal lacerations and hematomas (Table 13.4). Most renal injuries are treated conservatively, with surgical intervention reserved for collecting system disruptions and vascular injuries (Fig. 13.22). Delayed CT images are necessary to assess the integrity of the collecting system. Avulsion of the renal pedicle, characterized on CT as very poor or absent enhancement of the kidney, carries a high risk of renal devascularization. Rupture of the urinary bladder is a complication of pelvic trauma. Patients with gross hematuria or pelvic fractures should undergo CT cystography. Intraperitoneal rupture requires surgical repair, whereas extraperitoneal rupture can be treated conservatively. However, there may be combined intraperitoneal and extraperitoneal bladder rupture in some trauma patients.

\subsubsection{Diaphragmatic Injuries}

Diaphragmatic injuries are caused by sudden increase in intra-abdominal pressure from blunt abdominal trauma. CT findings include diaphragmatic discontinuity, herniation of abdominal viscera into the thorax, constriction of herniated abdominal contents through a diaphragmatic defect, and dependent position of the herniated viscera along the posterior chest wall (dependent viscera sign). Multiplanar CT imaging can be helpful in identifying diaphragmatic injuries.

\subsubsection{Vascular Injuries}

Abdominal aortic injuries in high-speed motor vehicle collisions can be life-threatening. Obvious findings include large retroperitoneal hematoma and active extravasation. More subtle injuries include pseudoaneurysm, intimal flap, and thrombosis. Thoracic aortic transection usually requires emergency surgical repair, preferably utilizing endovascular techniques, but selected less severe aortic injuries can be watched closely initially without repair. Abdominal aortic injuries most commonly result from high-speed motor vehicle collisions in which the aorta is compressed between a lap belt and the lumbar spine. It is important to note that a substantial amount of blood loss in the retroperitoneum may be clinically occult and will not be detected with FAST. Extraperitoneal hemorrhage in the pelvis often is 

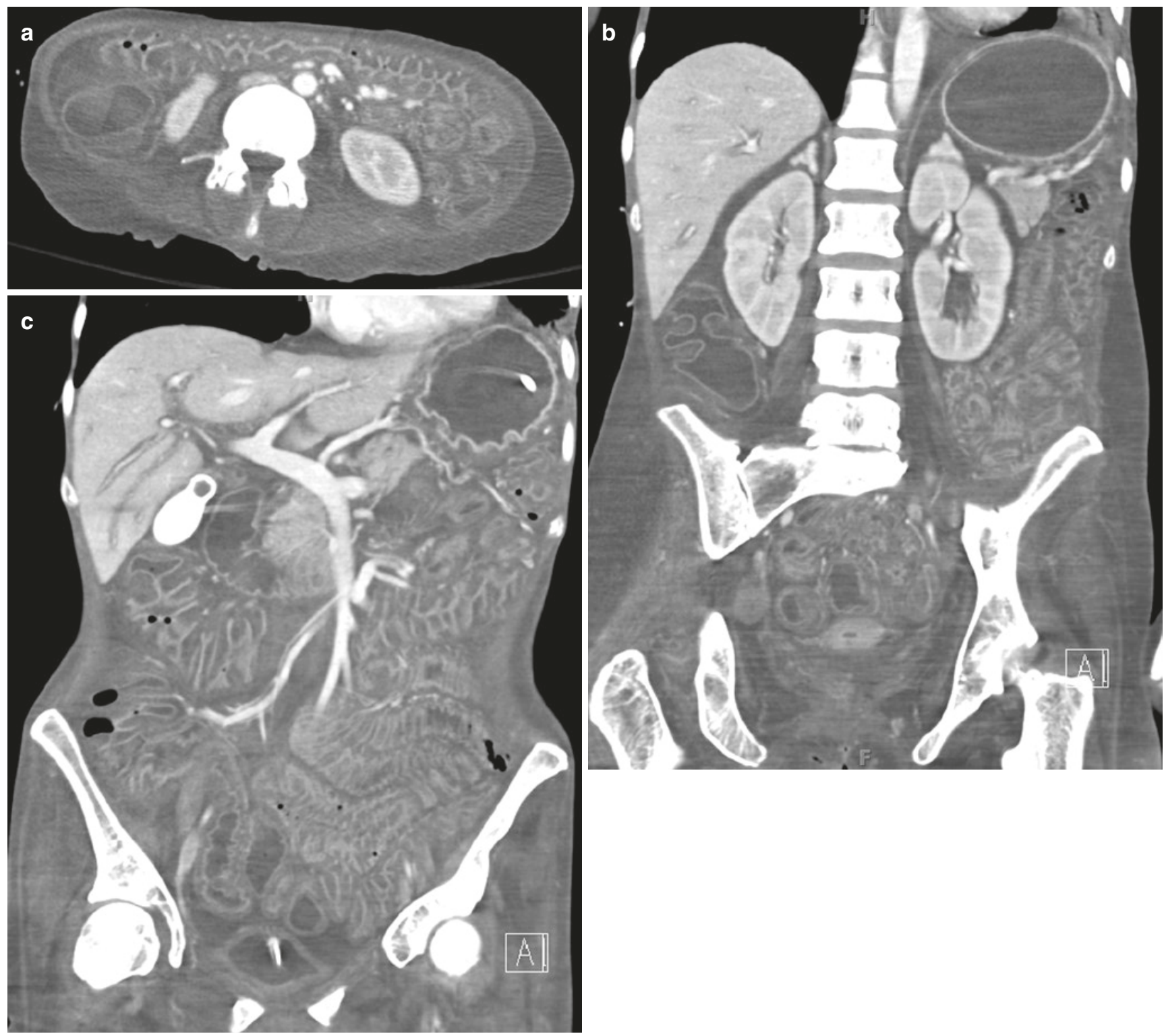

Fig. 13.20 Hypoperfusion complex. 42-year-old woman with acute blood loss. Transaxial (a) and coronal (b, c) contrast-enhanced CT images show diffuse edema, bowel wall thickening, collapse of the inferior vena cava, and hyperenhancement of the adrenal glands

associated with pelvic fractures. CT angiography is useful in this setting to evaluate for active extravasation, which can be treated in most cases with endovascular embolization.

\subsubsection{Penetrating Abdominal Trauma}

The need for surgery after a stab wound depends upon the location and depth of penetration. Wounds in any location that penetrate the peritoneum require surgical exploration because of the likelihood of bowel injury. Anterior wounds that penetrate the deep fascia usually require laparoscopy or laparotomy because of the possibility of bowel injury. In contradistinction, posterior stab wounds that penetrate the deep muscular fascia may be confined to the paraspinal muscles and may not be deep enough to penetrate the peritoneum, thus not necessitating surgical exploration. CT with intravenous, oral, and rectal contrast is useful in determining the depth of wound penetration and in identifying organ injury, retroperitoneal hematoma, and hemoperitoneum.

Gunshot wounds are much more complex injuries that often are difficult to evaluate clinically. If clinical examination 
or radiographs indicate that the bullet might have crossed bowel, laparoscopy or exploratory laparotomy is indicated, and CT is not necessary. Similarly, patients who are hemodynamically unstable require surgical exploration. In the remainder of patients who are hemodynamically stable, CT is

Table 13.4 American Association for the Surgery of Trauma (AAST) Kidney Injury Scale

\begin{tabular}{l|l}
$\begin{array}{l}\text { Injury grade } \\
\text { and type }\end{array}$ & Description \\
\hline Contusion & $\begin{array}{l}\text { Microscopic or gross hematuria, with normal } \\
\text { urologic studies }\end{array}$ \\
\hline Hematoma & $\begin{array}{l}\text { Subcapsular nonexpanding, without parenchymal } \\
\text { laceration }\end{array}$ \\
\hline II & $\begin{array}{l}\text { Nonexpanding perirenal hematoma confined to } \\
\text { renal retroperitoneum } \\
\text { Hematoma }\end{array}$ \\
\hline Laceration & $\begin{array}{l}<1-c m \text { parenchymal depth of renal cortex, without } \\
\text { urinary extravasation }\end{array}$ \\
\hline III & $\begin{array}{l}>1 \text {-cm parenchymal depth of renal cortex, without } \\
\text { collecting system rupture or urinary extravasation }\end{array}$ \\
\hline Laceration & $\begin{array}{l}\text { Parenchymal laceration extending through renal } \\
\text { cortex, medulla, and collecting system }\end{array}$ \\
\hline IV & $\begin{array}{l}\text { Main renal artery or vein injury, with contained } \\
\text { hemorrhage }\end{array}$ \\
\hline Laceration & Completely shattered kidney \\
\hline Vascular & $\begin{array}{l}\text { Avulsion of renal hilum, which devascularizes the } \\
\text { kidney }\end{array}$ \\
\hline$V$ & Laceration
\end{tabular}

Adapted from Moore et al. (1989), with permission

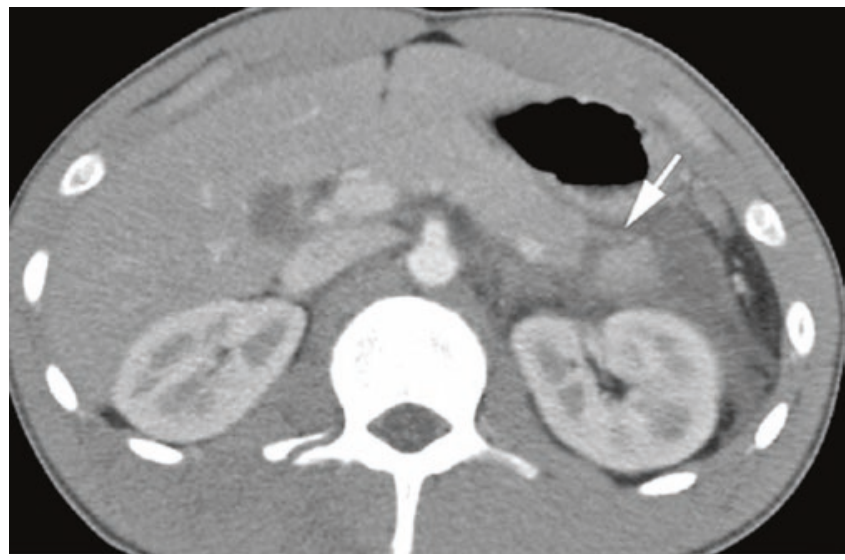

Fig. 13.21 Pancreatic laceration. Transaxial CT image demonstrates a linear hypoattenuating defect (arrow) extending through the tail of the pancreas. The pancreatic tail is surrounded by fluid effective in delineating the path of the bullet and identifying solid and hollow organ injuries. Solid organ injuries are staged using the AAST criteria. Findings of bowel perforation including pneumoperitoneum and extraluminal leakage of orally or rectally administered contrast material require surgical exploration; however, small perforations of bowel may be very difficult to diagnose with CT. If CT demonstrates peritoneal fluid or the bullet path crossing bowel in the absence of pneumoperitoneum or contrast leakage, surgical exploration is indicated.
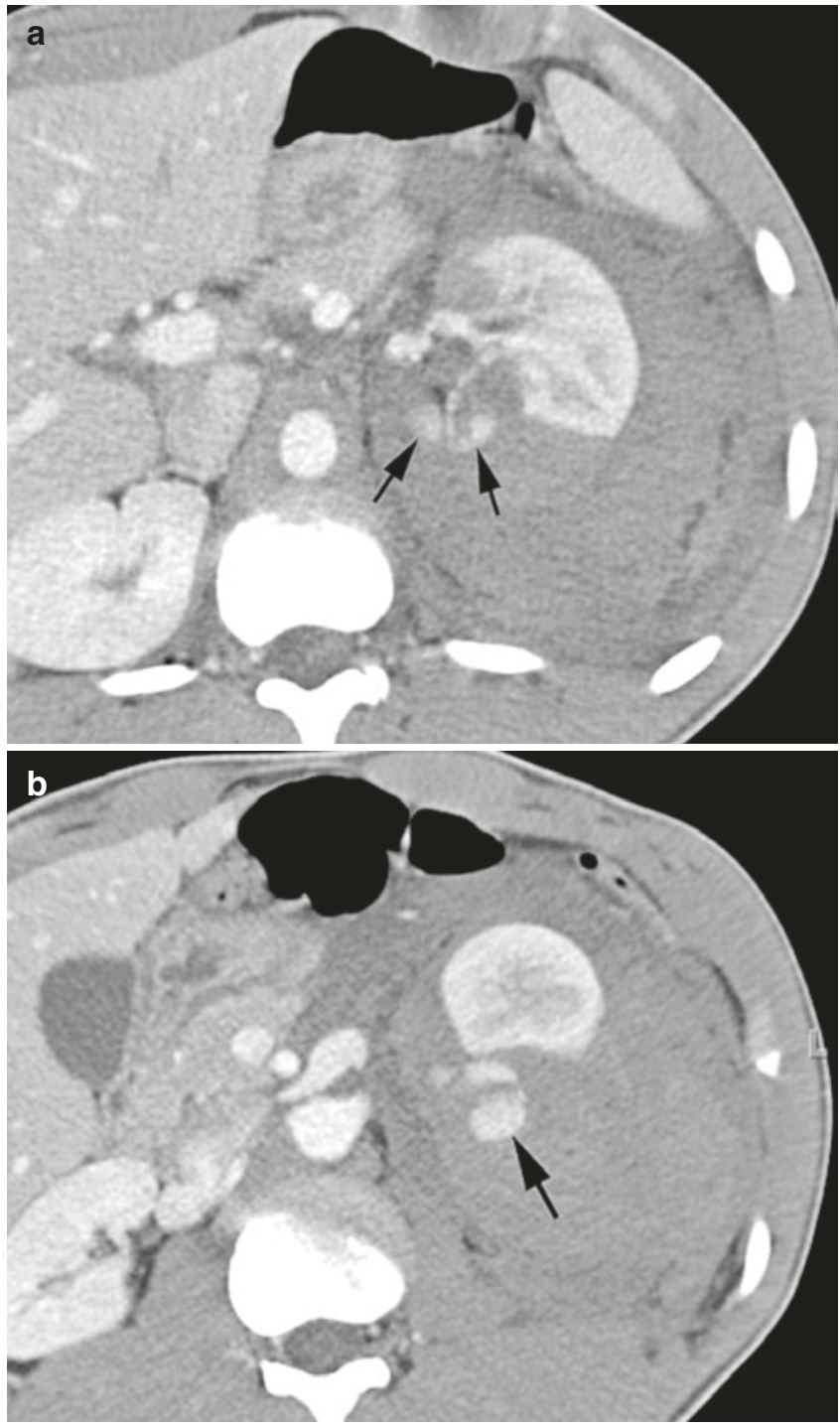

Fig. 13.22 (a, b) Deep (grade III) renal laceration with active extravasation. Transaxial CT images (a, b) show a deep laceration of the left kidney that extends to the renal hilum. Arrows indicate active extravasation. A large hematoma surrounds the kidney and displaces it anteriorly 


\section{Take-Home Messages}

- Imaging of non-traumatic abdominal and pelvic pain, as well as imaging of acute abdominal and pelvic trauma, continues to evolve.

- CT, using a variety of protocols, remains the mainstay for imaging acute traumatic as well as nontraumatic conditions, especially in adults, although US frequently is used as the first examination in children and in the non-trauma setting, with subsequent CT utilized in children when necessary.

- MRI has a growing role, especially for follow-up examinations in the non-trauma setting and for initial imaging or following US in pregnant patients and in pediatric patients, to reduce radiation exposure.

- Close cooperation with the referring physician immediately prior to imaging remains essential for rapid and accurate diagnosis, as the character and location of the patient's pain, and other considerations, strongly influence the specific imaging examination(s) performed and the protocol(s) utilized, as well as the resulting imaging differential diagnosis.

\section{Suggested Reading}

Ahn SH, Mayo-Smith WW, Murphy BL, Reinert SE, Cronan JJ. Acute nontraumatic abdominal pain in adult patients: abdominal radiography compared with CT evaluation. Radiology. 2002;225:159-64.

Anderson SW, Soto JA, Lucey BC, et al. Abdominal 64-MDCT for suspected appendicitis: the use of oral and IV contrast material versus IV contrast material only. AJR. 2009;193:1282-8.

Atri M, Hanson JM, Grinblat L, et al. Surgically important bowel and/ or mesenteric injury in blunt trauma: accuracy of multidetector CT evaluation. Radiology. 2008;249:524-33.

Balthazar EJ, Robinson DL, Megibow AJ, Ranson JH. Acute pancreatitis: value of $\mathrm{CT}$ in establishing prognosis. Radiology. 1990;174:331-6.

Boscak AR, Shanmuganathan K, Mirvis SE, et al. Optimizing trauma multidetector CT protocol for blunt splenic injury: need for arterial and portal venous phase scans. Radiology. 2013;268:79-88.

Desir A, Ghaye B. CT of blunt diaphragmatic rupture. Radiographics. 2012;32:477-98.

Dewhurst C, Beddy P, Pedrosa I. MRI evaluation of acute appendicitis in pregnancy. J Magn Reson Imaging. 2013;37:566-75.

Drasin TE, Anderson SW, Asandra A, et al. MDCT evaluation of blunt abdominal trauma: clinical significance of free intraperitoneal fluid in males with absence of identifiable injury. AJR. 2001;191:1821-6.

Duke E, Kalb B, Arif-Tiwari H, et al. A systematic review and metaanalysis of diagnostic performance of MRI for evaluation of acute appendicitis. AJR. 2016;206:508-17.

Fuks D, Mouly C, Robert B, Hajji H, Yzet T, Regimbeau JM. Acute cholecystitis: preoperative CT can help the surgeon consider conversion from laparoscopic to open cholecystectomy. Radiology. 2012;263:128-38.

Gans B, Sodickson A. Imaging of blunt bowel, mesenteric, and body wall trauma. Semin Roentgenol. 2016;51:230-8.

Gordon RW, Anderson SW, Ozonoff A, et al. Blunt pancreatic trauma: evaluation with MDCT technology. Emerg Radiol. 2013;20:259-66.
Gore RM, Miller FH, Pereles FS, Yaghmai V, Berlin JW. Helical CT in the evaluation of the acute abdomen. AJR. 2000;174:901-13.

Hines J, Rosenfeld J, Duncan DR, Friedman B, Katz DS. Perforation of the mesenteric small bowel: etiologies and CT findings. Emerg Radiol. 2013;20:155-61.

Katz DS, Klein MA, Ganson G, Hines JJ. Imaging of abdominal pain in pregnancy. Radiol Clin North Am. 2012;50:149-71.

Katz DS, Khalid M, Coronel EE, Mazzie JP. Computed tomography imaging of the acute pelvis in females. Can Assoc Radiol J. 2013a;64:108-18.

Katz DS, Scheirey CD, Bordia R, Hines JJ, Javors BR, Scholz FJ. Computed tomography of miscellaneous regional and diffuse small bowel disorders. Radiol Clin North Am. 2013b;51:45-68.

Kereshi B, Lee KS, Siewert B, Mortele KJ. Clinical utility of magnetic resonance imaging in the evaluation of pregnant females with suspected acute appendicitis. Abdom Radiol. 2017 (published online: 28 August 2017).

Keyzer C, Cullus P, Tack D, De Maertelaer V, Bohy P, Gevenois PA. MDCT for suspected acute appendicitis in adults: impact of oral and IV contrast media at standard-dose and simulated low-dose techniques. AJR. 2009;193:1272-81.

LeBedis CA, Anderson SW, Soto JA. CT imaging of blunt traumatic bowel and mesenteric injuries. Radiol Clin North Am. 2012;50:123-36.

Lee SS, Park SH. Computed tomography evaluation of gastrointestinal bleeding and acute mesenteric ischemia. Radiol Clin North Am. 2013;51:29-43.

Levenson RB, Camacho MA, Horn E, Saghir A, McGillicuddy D, Sanchez LD. Eliminating routine oral contrast use for CT in the emergency department: impact on patient throughput and diagnosis. Emerg Radiol. 2012;19:513-7.

Lucey BC, Varghese JC, Anderson SW, Soto JA. Spontaneous hemoperitoneum: a bloody mess. Emerg Radiol. 2006;14:65-75.

MacKersie AB, Lane MJ, Gerhardt RT, et al. Nontraumatic acute abdominal pain: unenhanced helical CT compared with three-view acute abdominal series. Radiology. 2005;237:114-22.

Marmery H, Shanmuganathan K, Alexander MT, Mirvis SE. Optimization of selection of nonoperative management of blunt splenic injury: comparison of MDCT grading systems. AJR. 2007;189:1421-7.

Mellnick VM, McDowell C, Lubner M, et al. CT features of blunt abdominal aortic injury. Emerg Radiol. 2012;19:301-7.

Mirvis SE, Shanmuganagthan K. Imaging hemidiaphragmatic injury. Eur Radiol. 2007;17:1411-21.

Moore EE, Shackford SR, Pachter HL, et al. Organ injury scaling: spleen, liver, and kidney. J Trauma. 1989;29:1664-6.

Moore EE, Cogbill THJ, Jurkovich GJ, et al. J Trauma. 1995;38:323-4, with permission from Lippincott Williams and Wilkins/Wolter Kluwer Health.

Orwig D, Federle MP. Localized clotted blood as evidence of visceral trauma on CT: the sentinel clot sign. AJR. 1989;153:747-9.

Pooler BD, Lawrence EM, Pickhardt PJ. Alternative diagnoses to suspected appendicitis at CT. Radiology. 2012;265:733-42.

Rapp EJ, Naim F, Kadivar K, Davarpanah A, Cornfeld D. Integrating MR imaging into the clinical workup of pregnant patients suspected of having appendicitis is associated with a lower negative laparotomy rate: a single-institution study. Radiology. 2013;267:137-44.

Revzin MV, Mathur M, Dave HB, Macer ML, Spektor M. Pelvic inflammatory disease: multi-modality imaging approach with clinical-pathologic correlation. Radiographics. 2016;36:1579-96.

Robinson JD, Sandstrom CK, Lehnert BE, Gross JA. Imaging of blunt abdominal solid organ trauma. Semin Roentgenol. 2016;51:215-29.

Rosen MP, Ding A, Blake MA, et al. ACR Appropriateness Criteria right lower quadrant pain - suspected appendicitis. JACR. 2011;8:749-55.

Santillan CS. Computed tomography of small bowel obstruction. Radiol Clin North Am. 2013;51:17-27.

Shakespear JS, Shaaban AM, Rezvani M. CT findings of acute cholecystitis and its complications. AJR. 2010;194:1523-9.

Singh AK, Gervais DA, Hahn PF, Sagar P, Muller PR, Novelline R. Acute epiploic appendagitis and its mimics. Radiographics. 2005;25:1521-34. 
Smith RC, Varanelli M. Diagnosis and management of acute ureterolithiasis. AJR. 2000;175:3-6.

Soto JA, Anterson SW. Multidetector CT of blunt abdominal trauma. Radiology. 2012;265:678-93.

Stojer J, van Randen A, Lameris W, Boermeester MA. Imaging patients with acute abdominal pain. Radiology. 2009;253:31-46.

Stuhlfaut JW, Lucey BC, Varghese JC, Soto JA. Blunt abdominal trauma: utility of 5-minute delayed $\mathrm{CT}$ with a reduced radiation dose. Radiology. 2006;238:473-9.

Thoeni RF. The revised Atlanta classification of acute pancreatitis: its importance for the radiologist and its effect on treatment. Radiology. 2012;262:751-64.
Werner A, Diehl SJ, Farag-Soliman M, Duber C. Multi-slice spiral CT in routine diagnosis of suspected acute left-sided colonic diverticulitis: a prospective study of 120 patients. Eur Radiol. 2003;13:2596-603.

Wieseler KM, Bhargava P, Kanal KM, Vaidya S, Stewart BK, Dighe MK. Imaging in pregnant patients: examination appropriateness. Radiographics. 2010;30:1215-29.

Yu J, Fulcher AS, Wang DB, et al. Frequency and importance of small amount of isolated pelvic free fluid detected with multidetector CT in male patients with blunt trauma. Radiology. 2010;256:799-805.

Open Access This chapter is licensed under the terms of the Creative Commons Attribution 4.0 International License (http://creativecommons.org/ licenses/by/4.0/), which permits use, sharing, adaptation, distribution and reproduction in any medium or format, as long as you give appropriate credit to the original author(s) and the source, provide a link to the Creative Commons license and indicate if changes were made.

The images or other third party material in this book are included in the book's Creative Commons license, unless indicated otherwise in a credit line to the material. If material is not included in the book's Creative Commons license and your intended use is not permitted by statutory regulation or exceeds the permitted use, you will need to obtain permission directly from the copyright holder. 